\title{
Spectral estimates for resolvent differences of self-adjoint elliptic operators
}

\author{
Jussi Behrndt, Matthias Langer and Vladimir Lotoreichik
}

\begin{abstract}
The concept of quasi boundary triples and Weyl functions from extension theory of symmetric operators in Hilbert spaces is developed further and spectral estimates for resolvent differences of two self-adjoint extensions in terms of general operator ideals are proved. The abstract results are applied to self-adjoint realizations of second order elliptic differential operators on bounded and exterior domains, and partial differential operators with $\delta$-potentials supported on hypersurfaces are studied.
\end{abstract}

Mathematics Subject Classification (2010). 35P05, 35P20, 47F05, 47L20, 81Q10, 81Q15.

Keywords. Elliptic operator, self-adjoint extension, operator ideal, $\delta$ potential, quasi boundary triple, Weyl function.

\section{Introduction}

The concept of boundary triples and Weyl functions is an efficient tool for the description and analysis of the self-adjoint extensions of a given symmetric operator $A$ in a Hilbert space $\mathcal{H}$. Roughly speaking, two boundary mappings $\Gamma_{0}, \Gamma_{1}$ are used, which are defined on the domain of the adjoint $A^{*}$, map into an auxiliary Hilbert space $\mathcal{G}$ (the space of boundary values) and satisfy an abstract Green identity

$$
\left(A^{*} f, g\right)_{\mathcal{H}}-\left(f, A^{*} g\right)_{\mathcal{H}}=\left(\Gamma_{1} f, \Gamma_{0} g\right)_{\mathcal{G}}-\left(\Gamma_{0} f, \Gamma_{1} g\right)_{\mathcal{G}}
$$

for all $f, g \in \operatorname{dom} A^{*}$. The self-adjoint extensions $A_{\Theta}$ are characterized as restrictions of $A^{*}$ to the set of elements $f \in \operatorname{dom} A^{*}$ satisfying the abstract boundary condition

$$
\left(\begin{array}{c}
\Gamma_{0} f \\
\Gamma_{1} f
\end{array}\right) \in \Theta
$$

where $\Theta$ is a self-adjoint linear operator or relation in $\mathcal{G}$. Boundary triples were successfully applied in many situations, in particular, ordinary differential operators; see, e.g. [15, 19, 23, 25, 48]. For second order differential 
operators on an interval one usually chooses $\Gamma_{0}$ to assign Dirichlet data and $\Gamma_{1}$ to assign Neumann data at the endpoints of the interval, or vice versa.

For elliptic partial differential operators the same approach with the boundary mappings $\Gamma_{0}$ and $\Gamma_{1}$ as the Dirichlet trace and the conormal derivative, respectively, leads to serious difficulties since Green's identity does not make sense on the whole domain of the maximal operator. Based on ideas from $[39,64]$ a boundary triple with the trace and a regularized version of the conormal derivatives was used for elliptic operators in [20, 21, 52]. However, in order to work with the usual trace and conormal derivative, a generalization of the notion of boundary triples was introduced in [10]: quasi boundary triples. In this setting the boundary mappings $\Gamma_{0}$ and $\Gamma_{1}$ are not defined on the whole domain of the maximal operator $A^{*}$ but only on the domain of some restriction $T$ whose closure is $A^{*}$; the abstract Green identity (1.1) holds then with $A^{*}$ replaced by $T$. For elliptic operators on a bounded domain $\Omega$ one can choose $T$, for instance, to be defined on $H^{2}(\Omega)$. The aim of the current paper is to develop the theory of quasi boundary triples further and use it to prove new results in spectral theory. We apply the abstract results to elliptic operators on bounded and exterior domains and to partial differential operators with $\delta$-potentials supported on hypersurfaces in $\mathbb{R}^{n}$, see also $[11,13]$.

In the following, let $A$ be a symmetric operator in a Hilbert space $\mathcal{H}$ and let $\left\{\mathcal{G}, \Gamma_{0}, \Gamma_{1}\right\}$ be a quasi boundary triple for $A^{*}=\bar{T}$, with $A_{0}=T$ 个 $\operatorname{ker} \Gamma_{0}$ self-adjoint, see also Definition 3.1. A very important object that is associated with a quasi boundary triple is the Weyl function $M$, which, for $\lambda \in \rho\left(A_{0}\right)$ is an operator in $\mathcal{G}$ that satisfies $\Gamma_{1} f_{\lambda}=M(\lambda) \Gamma_{0} f_{\lambda}$ for $f_{\lambda} \in$ $\operatorname{ker}(T-\lambda)$. In our treatment of elliptic operators in Sections 4.1 and 4.2 it will turn out that $M(\lambda)$ is the Neumann-to-Dirichlet map. In the quasi boundary triple setting a self-adjoint relation $\Theta$ in $\mathcal{G}$ as abstract boundary condition in (1.2) does not automatically induce a self-adjoint restriction $A_{\Theta}$ of $T$ in $\mathcal{H}$ (as is the case for boundary triples) but only a symmetric operator $A_{\Theta}$. In Theorem 3.11 we provide a sufficient condition on the Weyl function $M(\lambda)$ and $\Theta$ so that the operator $A_{\Theta}$ becomes self-adjoint. Applied to elliptic operators, this theorem yields a wide class of local and non-local boundary conditions for which there exists a self-adjoint realization in an $H^{2}$-setting (Theorem 4.5 and Corollary 4.6). The proof of Theorem 3.11 uses a variant of Krein's formula, in which the resolvents of $A_{\Theta}$ and $A_{0}$ are compared, see Theorem 3.8 and [11, Theorem 6.16]. Krein's formula is also an important ingredient in the proofs of the results of the core Section 3.3 in the abstract part of the present paper. There we prove spectral estimates for resolvent differences of two self-adjoint extensions $A_{\Theta_{1}}$ and $A_{\Theta_{2}}$ in terms of general operator ideals. This yields, in particular, spectral estimates of Schattenvon Neumann type. 
As mentioned above, we apply our abstract results to elliptic partial differential operators associated with the expression

$$
\mathcal{L}=-\sum_{j, k=1}^{n} \frac{\partial}{\partial x_{j}} a_{j k} \frac{\partial}{\partial x_{k}}+a
$$

on a domain $\Omega$ in $\mathbb{R}^{n}$ with compact $C^{\infty}$-boundary $\partial \Omega$. The domain $\Omega$ itself is allowed to be either bounded or the complement of a bounded set. We define the associated operator $T$ on $H^{2}(\Omega)$ if $\Omega$ is bounded, and on a set of functions which are in $H^{2}$ in a neighbourhood of $\partial \Omega$ if $\Omega$ is unbounded; for details see Definition 4.1. For the space of boundary values $\mathcal{G}$ we choose $L^{2}(\partial \Omega)$, and the boundary mappings are defined by

$$
\Gamma_{0} f=\left.\frac{\partial f}{\partial \nu_{\mathcal{L}}}\right|_{\partial \Omega}:=\left.\sum_{j, k=1}^{n} a_{j k} \nu_{j} \frac{\partial f}{\partial x_{k}}\right|_{\partial \Omega} \quad \text { and } \quad \Gamma_{1} f=\left.f\right|_{\partial \Omega},
$$

where $\nu(x)=\left(\nu_{1}(x), \ldots, \nu_{n}(x)\right)^{\top}$ is the unit vector at the point $x \in \partial \Omega$ pointing out of $\Omega$. After having established that $\left\{L^{2}(\partial \Omega), \Gamma_{0}, \Gamma_{1}\right\}$ is a quasi boundary triple (which is done in Theorem 4.2), we apply our abstract results from Section 3. In Theorem 4.5 we prove that, for an arbitrary bounded selfadjoint operator $B$ in $L^{2}(\partial \Omega)$ that satisfies $B\left(H^{1}(\partial \Omega)\right) \subset H^{1 / 2}(\partial \Omega)$, the elliptic expression $\mathcal{L}$ together with the boundary condition

$$
B\left(\left.f\right|_{\partial \Omega}\right)=\left.\frac{\partial f}{\partial \nu_{\mathcal{L}}}\right|_{\partial \Omega}
$$

gives rise to a self-adjoint operator $L^{2}(\Omega)$ whose domain consists of functions $f$ which are in $H^{2}$ in a neighbourhood of the boundary $\partial \Omega$. The boundary condition in (1.4) corresponds to the abstract boundary condition (1.2) with $\Theta=B^{-1}$ and contains a large class of Robin boundary conditions but also non-local boundary conditions.

In order to describe our main results on spectral estimates of resolvent differences of elliptic operators, we use the following notation here in the introduction. We write

$$
H_{1} \stackrel{r}{-} H_{2},
$$

if the singular values $s_{k}$ of the resolvent difference $\left(H_{1}-\lambda\right)^{-1}-\left(H_{2}-\lambda\right)^{-1}$ of two self-adjoint operators $H_{1}, H_{2}$ satisfy $s_{k}=\mathrm{O}\left(k^{-1 / r}\right), k \rightarrow \infty$. In Theorem 4.10 we conclude from the abstract results in Section 3.3 that

$$
A_{\mathrm{N}} \frac{\frac{n-1}{3}}{-} A_{\Theta},
$$

where $A_{\mathrm{N}}$ is the Neumann realization of $\mathcal{L}$ and $\Theta$ is a self-adjoint relation in $L^{2}(\partial \Omega)$ so that $0 \notin \sigma_{\text {ess }}(\Theta)$ and $A_{\Theta}$ is self-adjoint. For instance, $\Theta=B^{-1}$ with a bounded self-adjoint $B$ as above, i.e. the partial differential operator with boundary condition (1.4), leads to (1.5). A slightly weaker estimate for the Laplacian on bounded domains was proved in [12]. M. Sh. Birman [16] proved that

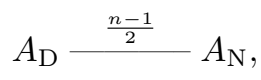


and later M.Sh. Birman and M.Z. Solomjak [17] and G. Grubb [40, 41] further investigated this relation and obtained the exact spectral asymptotics of the resolvent difference. We also refer to [43] for spectral asymptotics in the case of classical Robin boundary conditions. In general, the operator $A_{\Theta}$ as above is closer to the Neumann operator $A_{\mathrm{N}}$ in the sense of (1.5) than to the Dirichlet operator $A_{\mathrm{D}}$. We can compare also two operators with non-local boundary conditions $A_{\Theta_{1}}, A_{\Theta_{2}}$ under some assumption on $\Theta_{1}-\Theta_{2}$, namely in Theorem 4.15 if $s_{k}\left(\Theta_{1}-\Theta_{2}\right)=\mathrm{O}\left(k^{-1 / r}\right), k \rightarrow \infty$, then

$$
A_{\Theta_{1}} \frac{\frac{r(n-1)}{3 r+n-1}}{3 A_{\Theta_{2}}} \text {. }
$$

We also apply our abstract results to elliptic operators on $\mathbb{R}^{n}$ with a $\delta$-potential supported on a bounded $C^{\infty}$-hypersurface $\Sigma$, which splits $\mathbb{R}^{n}$ into a bounded interior domain $\Omega_{\mathrm{i}}$ and an exterior domain $\Omega_{\mathrm{e}}$; cf. [13] for a more detailed treatment of the case $\mathcal{L}=-\Delta+V$. The spectral theory of Schrödinger operators with $\delta$-potentials on surfaces has been developed since the late 1980s; see, e.g. the papers [5, 18, 29, 30, 31]. In Section 4.3 we use quasi boundary triples to construct a self-adjoint differential operator $A_{\delta, \alpha}$ in $L^{2}\left(\mathbb{R}^{n}\right)$ associated with $\mathcal{L}$ in (1.3) and interface conditions

$$
\left.f_{\mathrm{e}}\right|_{\Sigma}=\left.f_{\mathrm{i}}\right|_{\Sigma},\left.\quad \frac{\partial f_{\mathrm{i}}}{\partial \nu_{\mathcal{L}_{\mathrm{i}}}}\right|_{\Sigma}+\left.\frac{\partial f_{\mathrm{e}}}{\partial \nu_{\mathcal{L}_{\mathrm{e}}}}\right|_{\Sigma}=\left.\alpha f\right|_{\Sigma},
$$

where $f_{\mathrm{i}}$ and $f_{\mathrm{e}}$ are the restrictions of $f$ to $\Omega_{\mathrm{i}}$ and $\Omega_{\mathrm{e}}$, and $\alpha$ is a real-valued function in $C^{1}(\Sigma)$. The operator $A_{\delta, \alpha}$ can be interpreted as a differential operator with a $\delta$-potential of strength $\alpha$. Using our abstract results on resolvent differences we then conclude

$$
A_{\text {free }} \frac{\frac{n-1}{3}}{} A_{\delta, \alpha} .
$$

We mention that, independently, V. Ryzhov developed a concept that has similarities to the concept of quasi boundary triples in [61, 62]. Moreover, for extension theory of elliptic operators on non-smooth domains and Dirichlet-to-Neumann maps we refer to the recent contributions $[1,6,33,34$, $35,59]$. Let us also mention $[4,24,27,47,53,54,58,60]$ for other generalizations of boundary triples and their Weyl functions.

\section{Preliminaries}

\subsection{Notation and linear relations}

Throughout this paper let $(\mathcal{H},(\cdot, \cdot))$ and $(\mathcal{G},(\cdot, \cdot))$ be Hilbert spaces. In general, $\mathcal{H}$ and $\mathcal{G}$ are allowed to be non-separable, but in some theorems separability is assumed. The linear space of bounded linear operators defined on $\mathcal{H}$ with values in $\mathcal{G}$ is denoted by $\mathcal{B}(\mathcal{H}, \mathcal{G})$. If $\mathcal{H}=\mathcal{G}$, we simply write $\mathcal{B}(\mathcal{H})$. We shall often deal with (closed) linear relations in $\mathcal{H}$, that is, (closed) linear subspaces of $\mathcal{H} \oplus \mathcal{H}$. The set of closed linear relations in $\mathcal{H}$ is denoted by $\widetilde{\mathcal{C}}(\mathcal{H})$, and for elements in a relation we usually use a vector notation. Linear operators in $\mathcal{H}$ are viewed as linear relations via their graphs. The 
domain, range, kernel, multi-valued part and the inverse of a relation $T$ in $\mathcal{H}$ are denoted by $\operatorname{dom} T, \operatorname{ran} T, \operatorname{ker} T, \operatorname{mul} T$ and $T^{-1}$, respectively.

Let $T \in \widetilde{\mathcal{C}}(\mathcal{H})$ be a closed linear relation in $\mathcal{H}$. The resolvent set $\rho(T)$ of $T$ is the set of all $\lambda \in \mathbb{C}$ such that $(T-\lambda)^{-1} \in \mathcal{B}(\mathcal{H})$; the spectrum $\sigma(T)$ of $T$ is the complement of $\rho(T)$ in $\mathbb{C}$. A point $\lambda \in \mathbb{C}$ is an eigenvalue of a linear relation $T$ if $\operatorname{ker}(T-\lambda) \neq\{0\}$; in this case we write $\lambda \in \sigma_{\mathrm{p}}(T)$. For a linear relation $T$ in $\mathcal{H}$ the adjoint relation $T^{*}$ is a closed linear relation. A linear relation $S$ in $\mathcal{H}$ is said to be symmetric (self-adjoint) if $S \subset S^{*}$ ( $S=S^{*}$, respectively). Recall that a symmetric relation is self-adjoint if and only if $\operatorname{ran}\left(S-\lambda_{ \pm}\right)=\mathcal{H}$ holds for some $\lambda_{+} \in \mathbb{C}^{+}$and some $\lambda_{-} \in \mathbb{C}^{-}$, where $\mathbb{C}^{ \pm}:=\{z \in \mathbb{C}: \pm \operatorname{Im} z>0\}$; in this case we have $\operatorname{ran}(S-\lambda)=\mathcal{H}$ for all $\lambda \in \mathbb{C} \backslash \mathbb{R}$.

For a self-adjoint relation $S=S^{*}$ in $\mathcal{H}$ the multi-valued part mul $S$ is the orthogonal complement of $\operatorname{dom} S$ in $\mathcal{H}$. Setting $\mathcal{H}_{\text {op }}:=\overline{\operatorname{dom} S}$ and $\mathcal{H}_{\infty}=$ mul $S$ one verifies that $S$ can be written as the direct orthogonal sum $S=S_{\text {op }} \oplus S_{\infty}$ of an (in general unbounded) self-adjoint operator $S_{\text {op }}$ in the Hilbert space $\mathcal{H}_{\text {op }}$ and the "pure" relation $S_{\infty}=\left\{\left(\begin{array}{c}0 \\ f^{\prime}\end{array}\right): f^{\prime} \in \operatorname{mul} S\right\}$ in the Hilbert space $\mathcal{H}_{\infty}$; the corresponding space decomposition is $\mathcal{H}=\mathcal{H}_{\text {op }} \oplus \mathcal{H}_{\infty}$. We say that a point $\lambda \in \mathbb{R}$ belongs to the essential spectrum $\sigma_{\text {ess }}(S)$ of the self-adjoint relation $S$ if $\lambda \in \sigma_{\text {ess }}\left(S_{\text {op }}\right)$. The essential spectrum of a closed operator $T$ in $\mathcal{H}$ is the set of $\lambda \in \mathbb{C}$ such that $T-\lambda$ is not a Fredholm operator.

\subsection{Operator ideals and singular values}

In this section let $\mathcal{H}$ and $\mathcal{K}$ be separable Hilbert spaces. Denote by $\mathfrak{S}_{\infty}(\mathcal{H}, \mathcal{K})$ the closed subspace of compact operators in $\mathcal{B}(\mathcal{H}, \mathcal{K})$; if $\mathcal{H}=\mathcal{K}$, we simply write $\mathfrak{S}_{\infty}(\mathcal{H})$. We define classes of operator ideals along the lines of [57].

Definition 2.1. Suppose that, for every pair of Hilbert spaces $\mathcal{H}, \mathcal{K}$, we are given a subset $\mathfrak{A}(\mathcal{H}, \mathcal{K})$ of $\mathfrak{S}_{\infty}(\mathcal{H}, \mathcal{K})$. The set

$$
\mathfrak{A}:=\bigcup_{\mathcal{H}, \mathcal{K} \text { Hilbert spaces }} \mathfrak{A}(\mathcal{H}, \mathcal{K})
$$

is said to be a class of operator ideals if the following conditions are satisfied:

(i) the rank-one operators $x \mapsto(x, u) v$ are in $\mathfrak{A}(\mathcal{H}, \mathcal{K})$ for all $u \in \mathcal{H}, v \in \mathcal{K}$;

(ii) $A+B \in \mathfrak{A}(\mathcal{H}, \mathcal{K})$ for $A, B \in \mathfrak{A}(\mathcal{H}, \mathcal{K})$;

(iii) $C A B \in \mathfrak{A}\left(\mathcal{H}_{1}, \mathcal{K}_{1}\right)$ for $A \in \mathfrak{A}(\mathcal{H}, \mathcal{K}), B \in \mathcal{B}\left(\mathcal{H}_{1}, \mathcal{H}\right), C \in \mathcal{B}\left(\mathcal{K}, \mathcal{K}_{1}\right)$. Moreover, we write $\mathfrak{A}(\mathcal{H})$ for $\mathfrak{A}(\mathcal{H}, \mathcal{H})$.

If $\mathfrak{A}$ is a class of operator ideals, then the sets $\mathfrak{A}(\mathcal{H}, \mathcal{K})$ are two-sided operator ideals for every pair $\mathcal{H}, \mathcal{K}$; for the latter notion see also, e.g. [36, 56]. For two classes of operator ideals $\mathfrak{A}, \mathfrak{B}$ we define the product and the adjoint by

$$
\begin{aligned}
\mathfrak{A} \cdot \mathfrak{B} & :=\{T: \text { there exist } A \in \mathfrak{A}, B \in \mathfrak{B} \text { so that } T=A B\}, \\
\mathfrak{A}^{*} & :=\left\{A^{*}: A \in \mathfrak{A}\right\} .
\end{aligned}
$$


These sets are again classes of operator ideals; see [57]. The elements in the product $\mathfrak{A} \cdot \mathfrak{B}$ are denoted by $(\mathfrak{A} \cdot \mathfrak{B})(\mathcal{H}, \mathcal{K})$, so that

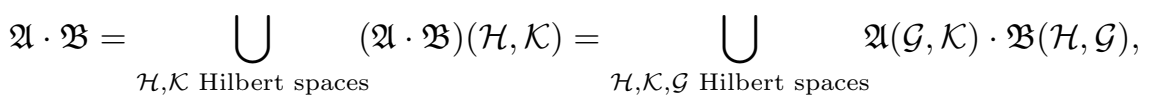

where the products $\mathfrak{A}(\mathcal{G}, \mathcal{K}) \cdot \mathfrak{B}(\mathcal{H}, \mathcal{G})$ are defined by

$$
\begin{aligned}
\mathfrak{A}(\mathcal{G}, \mathcal{K}) & \cdot \mathfrak{B}(\mathcal{H}, \mathcal{G}) \\
& :=\{T: \text { there exist } A \in \mathfrak{A}(\mathcal{G}, \mathcal{K}), B \in \mathfrak{B}(\mathcal{H}, \mathcal{G}) \text { so that } T=A B\} .
\end{aligned}
$$

Later also the notation $\mathfrak{A}^{*}(\mathcal{K}, \mathcal{H}):=\left\{A^{*}: A \in \mathfrak{A}(\mathcal{H}, \mathcal{K})\right\}$ will be used.

The next lemma is used to extend assertions about resolvent differences from one $\lambda$ to a bigger set of $\lambda$. For a special operator ideal, cf. [13, Lemma 2.2].

Lemma 2.2. Let $\mathfrak{A}$ be a class of operator ideals. Moreover, let $H$ and $K$ be closed linear relations in a separable Hilbert space $\mathcal{H}$. If

$$
(H-\lambda)^{-1}-(K-\lambda)^{-1} \in \mathfrak{A}(\mathcal{H})
$$

for some $\lambda \in \rho(H) \cap \rho(K)$, then (2.1) holds for all $\lambda \in \rho(H) \cap \rho(K)$.

Proof. Let $\lambda, \mu \in \rho(H) \cap \rho(K)$ and define

$$
E:=I+(\mu-\lambda)(H-\mu)^{-1}, \quad F:=I+(\mu-\lambda)(K-\mu)^{-1},
$$

which are both bounded operators in $\mathcal{H}$. The resolvent identity implies that

$$
E(H-\lambda)^{-1}=(H-\mu)^{-1} \text { and }(K-\lambda)^{-1} F=(K-\mu)^{-1} .
$$

Using this and the definition of $E, F$ one easily computes

$$
(H-\mu)^{-1}-(K-\mu)^{-1}=E\left((H-\lambda)^{-1}-(K-\lambda)^{-1}\right) F .
$$

Now the assertion follows from the ideal property of $\mathfrak{A}(\mathcal{H})$.

Recall that the singular values (or $s$-numbers) $s_{k}(A), k=1,2, \ldots$, of a compact operator $A \in \mathfrak{S}_{\infty}(\mathcal{H}, \mathcal{K})$ are defined as the eigenvalues $\lambda_{k}(|A|)$ of the non-negative compact operator $|A|=\left(A^{*} A\right)^{1 / 2} \in \mathfrak{S}_{\infty}(\mathcal{H})$, which are enumerated in non-increasing order and with multiplicities taken into account. Let $A \in \mathfrak{S}_{\infty}(\mathcal{H}, \mathcal{K})$ and assume that $\mathcal{H}$ and $\mathcal{K}$ are infinite dimensional Hilbert spaces. Then there exist orthonormal systems $\left\{\varphi_{1}, \varphi_{2}, \ldots\right\}$ and $\left\{\psi_{1}, \psi_{2}, \ldots\right\}$ in $\mathcal{H}$ and $\mathcal{K}$, respectively, such that $A$ admits the Schmidt expansion

$$
A=\sum_{k=1}^{\infty} s_{k}(A)\left(\cdot, \varphi_{k}\right) \psi_{k} .
$$

It follows, for instance, from (2.2) and the corresponding expansion for $A^{*} \in$ $\mathfrak{S}_{\infty}(\mathcal{K}, \mathcal{H})$ that the singular values of $A$ and $A^{*}$ coincide: $s_{k}(A)=s_{k}\left(A^{*}\right)$ for $k=1,2, \ldots$; see, e.g. [36, II. $\S 2.2]$. Moreover, if $\mathcal{G}$ and $\mathcal{L}$ are separable Hilbert spaces, $B \in \mathcal{B}(\mathcal{G}, \mathcal{H})$ and $C \in \mathcal{B}(\mathcal{K}, \mathcal{L})$, then the estimates

$$
s_{k}(A B) \leq\|B\| s_{k}(A) \quad \text { and } \quad s_{k}(C A) \leq\|C\| s_{k}(A), \quad k=1,2, \ldots,
$$


hold. If, in addition, $B \in \mathfrak{S}_{\infty}(\mathcal{G}, \mathcal{H})$, then

$$
s_{m+n-1}(A B) \leq s_{m}(A) s_{n}(B), \quad m, n=1,2 \ldots
$$

The proofs of the inequalities (2.3) and (2.4) are the same as in [36, II. $\$ 2.1$ and $\S 2.3]$, where these facts are shown for operators acting in the same space.

Recall that the Schatten-von Neumann ideals $\mathfrak{S}_{p}(\mathcal{H}, \mathcal{K})$ are defined by

$$
\mathfrak{S}_{p}(\mathcal{H}, \mathcal{K}):=\left\{A \in \mathfrak{S}_{\infty}(\mathcal{H}, \mathcal{K}): \sum_{k=1}^{\infty}\left(s_{k}(A)\right)^{p}<\infty\right\}, \quad p>0
$$

Besides the Schatten-von Neumann ideals also the operator ideals

$$
\begin{aligned}
& \mathfrak{S}_{p, \infty}(\mathcal{H}, \mathcal{K}):=\left\{A \in \mathfrak{S}_{\infty}(\mathcal{H}, \mathcal{K}): s_{k}(A)=\mathrm{O}\left(k^{-1 / p}\right), k \rightarrow \infty\right\}, \quad p>0, \\
& \mathfrak{S}_{p, \infty}^{(0)}(\mathcal{H}, \mathcal{K}):=\left\{A \in \mathfrak{S}_{\infty}(\mathcal{H}, \mathcal{K}): s_{k}(A)=\mathrm{o}\left(k^{-1 / p}\right), k \rightarrow \infty\right\},
\end{aligned}
$$

will play an important role later on. The sets

$\mathfrak{S}_{p}:=\bigcup_{\mathcal{H}, \mathcal{K}} \mathfrak{S}_{p}(\mathcal{H}, \mathcal{K}), \quad \mathfrak{S}_{p, \infty}:=\bigcup_{\mathcal{H}, \mathcal{K}} \mathfrak{S}_{p, \infty}(\mathcal{H}, \mathcal{K}), \quad \mathfrak{S}_{p, \infty}^{(0)}:=\bigcup_{\mathcal{H}, \mathcal{K}} \mathfrak{S}_{p, \infty}^{(0)}(\mathcal{H}, \mathcal{K})$

are classes of operator ideals in the sense of Definition 2.1.

We refer the reader to [36, III. $\S 7$ and III. $\S 14]$ for a detailed study of the classes $\mathfrak{S}_{p}, \mathfrak{S}_{p, \infty}$ and $\mathfrak{S}_{p, \infty}^{(0)}$. We list only some basic and well-known properties, which will be useful for us. It follows from $s_{k}(A)=s_{k}\left(A^{*}\right)$ that $\mathfrak{S}_{p}^{*}=\mathfrak{S}_{p}, \mathfrak{S}_{p, \infty}^{*}=\mathfrak{S}_{p, \infty}$ and $\left(\mathfrak{S}_{p, \infty}^{(0)}\right)^{*}=\mathfrak{S}_{p, \infty}^{(0)}$ hold.

Lemma 2.3. Let $p, q, r, s>0$ and assume that $\frac{1}{p}+\frac{1}{q}=\frac{1}{r}$. Then the following relations are true:

(i) $\mathfrak{S}_{p} \subset \mathfrak{S}_{p, \infty}^{(0)} \subset \mathfrak{S}_{p, \infty}$;

(ii) $\mathfrak{S}_{s, \infty} \subset \mathfrak{S}_{q}$ for all $q>s$;

(iii) $\mathfrak{S}_{p, \infty} \cdot \mathfrak{S}_{q, \infty}=\mathfrak{S}_{r, \infty}$;

(iv) $\mathfrak{S}_{p, \infty}^{(0)} \cdot \mathfrak{S}_{q, \infty}^{(0)}=\mathfrak{S}_{r, \infty}^{(0)}$;

(v) $\mathfrak{S}_{p} \cdot \mathfrak{S}_{q}=\mathfrak{S}_{r}$.

Proof. The first inclusion in (i) is a consequence of the fact that $\sum\left(s_{k}(A)\right)^{p}<$ $\infty$ implies $k\left(s_{k}(A)\right)^{p} \rightarrow 0$ for $k \rightarrow \infty$, and the second inclusion is clear. Assertion (ii) follows immediately from the definitions. In order to verify (iii) let $p, q>0$ and let $A \in \mathfrak{S}_{p, \infty}(\mathcal{H}, \mathcal{K})$ and $B \in \mathfrak{S}_{q, \infty}(\mathcal{G}, \mathcal{H})$, that is, the inequalities $s_{n}(A) \leq c_{a} n^{-1 / p}$ and $s_{n}(B) \leq c_{b} n^{-1 / q}, n \in \mathbb{N}$, hold with some constants $c_{a}, c_{b}>0$. From $(2.4)$ and $1 / p+1 / q=1 / r$ we obtain

$s_{2 n}(A B) \leq s_{2 n-1}(A B) \leq s_{n}(A) s_{n}(B) \leq \frac{c_{a} c_{b}}{n^{1 / p} n^{1 / q}}=\frac{2^{1 / r} c_{a} c_{b}}{(2 n)^{1 / r}} \leq \frac{2^{1 / r} c_{a} c_{b}}{(2 n-1)^{1 / r}}$,

which implies that $A B \in \mathfrak{S}_{r, \infty}(\mathcal{G}, \mathcal{K})$. In order to show equality, let $A \in$ $\mathfrak{S}_{r, \infty}(\mathcal{H}, \mathcal{K})$ and consider its Schmidt expansion $A=\sum_{k} s_{k}(A)\left(\cdot, \varphi_{k}\right) \psi_{k}$. Define operators $B: \mathcal{H} \rightarrow \mathcal{K}$ and $C: \mathcal{H} \rightarrow \mathcal{H}$ by

$$
B=\sum_{k}\left(s_{k}(A)\right)^{r / p}\left(\cdot, \varphi_{k}\right) \psi_{k}, \quad C=\sum_{k}\left(s_{k}(A)\right)^{r / q}\left(\cdot, \varphi_{k}\right) \varphi_{k} .
$$


The relations $A=B C, B \in \mathfrak{S}_{p, \infty}(\mathcal{H}, \mathcal{K}), C \in \mathfrak{S}_{q, \infty}(\mathcal{H}, \mathcal{H})$ show that $A \in$ $\mathfrak{S}_{p, \infty} \cdot \mathfrak{S}_{q, \infty}$. The same arguments as in (iii) can be used to show (iv). The inclusion " $\subset$ " in (v) follows from [36, III. $\S 7.2]$. The converse inclusion follows in a similar way as in (iii).

Sometimes we need also the notion of a symmetrically normed ideal: a two-sided ideal $\mathfrak{A}(\mathcal{H}, \mathcal{G})$ is a symmetrically normed ideal if it is a Banach space with respect to some norm $\|\cdot\|_{\mathfrak{A}}$ such that $\|C A B\|_{\mathfrak{A}} \leq\|C\|\|A\|_{\mathfrak{A}}\|B\|$ for $A \in \mathfrak{A}(\mathcal{H}, \mathcal{G}), B \in \mathcal{B}(\mathcal{H}), C \in \mathcal{B}(\mathcal{G})$ and $\|A\|_{\mathfrak{A}}=s_{1}(A)$ for rank one operators $A$; see [36, III. $\S 2.1$ and $\S 2.2]$. If a class of operator ideals consists of symmetrically normed ideals, then we call it a class of symmetrically normed ideals. The classes $\mathfrak{S}_{p}, \mathfrak{S}_{r, \infty}$ and $\mathfrak{S}_{r, \infty}^{(0)}$ are classes of symmetrically normed ideals for $p \geq 1$ and $r>1$; see [36, III. $\S 7$ and $\S 14]$.

The following lemma is needed in the proof of Proposition 3.5.

Lemma 2.4. Let $\mathfrak{A}(\mathcal{G})$ be a symmetrically normed ideal of $\mathcal{B}(\mathcal{G})$, let $C \in$ $\mathcal{B}(\mathcal{H})$ and assume that $A \in \mathfrak{A}(\mathcal{G})$ admits the factorization $A=B^{*} B$ with $B \in \mathcal{B}(\mathcal{G}, \mathcal{H})$. Then also $B^{*} C B \in \mathfrak{A}(\mathcal{G})$.

Proof. If $\mathcal{G}$ is finite-dimensional, then the assertion is trivial. So let us assume that $\mathcal{G}$ is infinite-dimensional. Observe first that $\left(s_{k}(A)\right)^{\frac{1}{2}}=s_{k}(B)=s_{k}\left(B^{*}\right)$ and $\lambda_{k}(A)=s_{k}(A)$ hold for all $k=1,2, \ldots$ Together with $(2.4)$ and the first inequality in $(2.3)$ we obtain

$$
s_{2 n}\left(B^{*} C B\right) \leq s_{2 n-1}\left(B^{*} C B\right) \leq s_{n}\left(B^{*}\right) s_{n}(C B) \leq\|C\| s_{n}(A)
$$

for $n=1,2, \ldots$ Let us write the non-negative compact operator $A \in \mathfrak{A}(\mathcal{G})$ in the form $A=\sum_{k=1}^{\infty} \lambda_{k}(A)\left(\cdot, \varphi_{k}\right) \varphi_{k}$ with an orthonormal basis $\left\{\varphi_{1}, \varphi_{2}, \ldots\right\}$ of eigenvectors corresponding to the eigenvalues $\lambda_{k}(A)$.

Define operators $V_{1}, V_{2} \in \mathcal{B}(\mathcal{G})$ by

$$
V_{1}:\left\{\begin{array}{rl}
\varphi_{2 k-1} & \mapsto \varphi_{k}, \\
\varphi_{2 k} & \mapsto 0,
\end{array} \quad V_{2}:\left\{\begin{array}{rl}
\varphi_{2 k-1} & \mapsto 0, \\
\varphi_{2 k} & \mapsto \varphi_{k},
\end{array} \quad k \in \mathbb{N} .\right.\right.
$$

Then the non-negative operator

$$
\widetilde{A}:=V_{1} A V_{1}^{*}+V_{2} A V_{2}^{*}=\sum_{k=1}^{\infty} \lambda_{k}(A)\left(\left(\cdot, \varphi_{2 k-1}\right) \varphi_{2 k-1}+\left(\cdot, \varphi_{2 k}\right) \varphi_{2 k}\right)
$$

belongs to $\mathfrak{A}(\mathcal{G})$, and its eigenvalues satisfy $\lambda_{2 n-1}(\widetilde{A})=\lambda_{2 n}(\widetilde{A})=\lambda_{n}(A)$. Hence we have $s_{k}\left(B^{*} C B\right) \leq\|C\| s_{k}(\widetilde{A}), k=1,2, \ldots$, and the claim follows from $[36$, III. $\S 2.2]$.

\section{Quasi boundary triples and Krein's formula}

\subsection{Quasi boundary triples, $\gamma$-fields and Weyl functions}

The notion of quasi boundary triples was introduced in connection with elliptic boundary value problems by the first two authors in [10] as a generalization of the notion of ordinary and generalized boundary triples from 
$[22,23,26,27,37,46,51]$. Let us start by recalling the basic definition from [10].

Definition 3.1. Let $(\mathcal{H},(\cdot, \cdot))$ be a Hilbert space and let $A$ be a closed symmetric relation in $\mathcal{H}$. We say that $\left\{\mathcal{G}, \Gamma_{0}, \Gamma_{1}\right\}$ is a quasi boundary triple for $A^{*}$ if $\Gamma_{0}$ and $\Gamma_{1}$ are linear mappings defined on a dense subspace $T$ of $A^{*}$ with values in the Hilbert space $(\mathcal{G},(\cdot, \cdot))$ such that $\Gamma:=\left(\begin{array}{c}\Gamma_{0} \\ \Gamma_{1}\end{array}\right): T \rightarrow \mathcal{G} \times \mathcal{G}$ has dense range, $\operatorname{ker} \Gamma_{0}$ is self-adjoint and the identity

$$
\left(f^{\prime}, g\right)-\left(f, g^{\prime}\right)=\left(\Gamma_{1} \hat{f}, \Gamma_{0} \hat{g}\right)-\left(\Gamma_{0} \hat{f}, \Gamma_{1} \hat{g}\right)
$$

holds for all $\hat{f}=\left(\begin{array}{c}f \\ f^{\prime}\end{array}\right), \hat{g}=\left(\begin{array}{c}g \\ g^{\prime}\end{array}\right) \in T$.

We recall some basic facts for quasi boundary triples, which can be found in [10]. Let $A$ be a closed symmetric relation in the Hilbert space $\mathcal{H}$. Then a quasi boundary triple for $A^{*}$ exists if and only if the deficiency indices $n_{ \pm}(A)=\operatorname{dim} \operatorname{ker}\left(A^{*} \mp i\right)$ of $A$ coincide. In the following, let $\left\{\mathcal{G}, \Gamma_{0}, \Gamma_{1}\right\}$ be a quasi boundary triple for $A^{*}$. Then $A$ coincides with $\operatorname{ker} \Gamma=\operatorname{ker} \Gamma_{0} \cap \operatorname{ker} \Gamma_{1}$ and $\Gamma=\left(\begin{array}{l}\Gamma_{0} \\ \Gamma_{1}\end{array}\right)$ regarded as a mapping from $\mathcal{H} \times \mathcal{H}$ into $\mathcal{G} \times \mathcal{G}$ is closable; cf. [10, Proposition 2.2]. Furthermore, as an immediate consequence of (3.1), the extension $A_{1}:=\operatorname{ker} \Gamma_{1}$ is a symmetric relation in $\mathcal{H}$.

Let $A$ be a closed symmetric relation in $\mathcal{H}$ and let $\left\{\mathcal{G}, \Gamma_{0}, \Gamma_{1}\right\}$ be a quasi boundary triple for $A^{*}$ with $T=\operatorname{dom} \Gamma$. We consider extensions of $A$ which are restrictions of $T$ defined by some abstract boundary condition. For a linear relation $\Theta \subset \mathcal{G} \times \mathcal{G}$ we define

$$
A_{\Theta}:=\{\hat{f} \in T: \Gamma \hat{f} \in \Theta\}=\Gamma^{-1}(\Theta) .
$$

If $\Theta \subset \mathcal{G} \times \mathcal{G}$ is an operator, then we have

$$
A_{\Theta}=\operatorname{ker}\left(\Gamma_{1}-\Theta \Gamma_{0}\right),
$$

and (3.3) holds also for linear relations $\Theta$ in $\mathcal{G}$ if the product and the difference on the right-hand side are understood in the sense of linear relations. Observe that the self-adjoint relation $A_{0}:=\operatorname{ker} \Gamma_{0}$ corresponds to the purely multivalued relation $\Theta=0^{-1}=\left\{\left(\begin{array}{l}0 \\ g\end{array}\right): g \in \mathcal{G}\right\}$ in $\mathcal{G}$. This little inconsistency in the notation should not lead to misunderstandings. It is not difficult to see that $\Theta \subset \Theta^{*}$ implies $A_{\Theta} \subset A_{\Theta}^{*}$. However, in contrast to ordinary boundary triples, self-adjointness of $\Theta$ does not imply self-adjointness or essential selfadjointness of $A_{\Theta}$; cf. [10, Proposition 4.11] for a counterexample, and see [10, Proposition 2.4] and Theorem 3.11 below for sufficient conditions.

In the following we set $\mathcal{G}_{0}:=\operatorname{ran} \Gamma_{0}$ and $\mathcal{G}_{1}:=\operatorname{ran} \Gamma_{1}$. Because $\operatorname{ran} \Gamma$ is dense in $\mathcal{G} \times \mathcal{G}$, it follows that $\mathcal{G}_{0}$ and $\mathcal{G}_{1}$ are dense subspaces of $\mathcal{G}$. Since $A_{0}:=$ $\operatorname{ker} \Gamma_{0} \subset T=\operatorname{dom} \Gamma$ is a self-adjoint extension of $A$ in $\mathcal{H}$, the decomposition

$$
T=A_{0} \widehat{+} \widehat{\mathcal{N}}_{\lambda, T}, \quad \widehat{\mathcal{N}}_{\lambda, T}:=\left\{\left(\begin{array}{c}
f_{\lambda} \\
\lambda f_{\lambda}
\end{array}\right): f_{\lambda} \in \mathcal{N}_{\lambda}(T):=\operatorname{ker}(T-\lambda)\right\},
$$

holds for all $\lambda \in \rho\left(A_{0}\right)$. Here $\widehat{+}$ denotes the direct sum of the subspaces $A_{0}$ and $\widehat{\mathcal{N}}_{\lambda, T}$ in $\mathcal{H} \oplus \mathcal{H}$. It follows that the mapping

$$
\left(\Gamma_{0} \uparrow \widehat{\mathcal{N}}_{\lambda, T}\right)^{-1}: \mathcal{G}_{0} \rightarrow \widehat{\mathcal{N}}_{\lambda, T}, \quad \lambda \in \rho\left(A_{0}\right),
$$


is well defined and bijective. Denote the orthogonal projection in $\mathcal{H} \oplus \mathcal{H}$ onto the first component of $\mathcal{H} \oplus \mathcal{H}$ by $\pi_{1}$.

Definition 3.2. Let $A$ be a closed symmetric relation in $\mathcal{H}$ and let $\left\{\mathcal{G}, \Gamma_{0}, \Gamma_{1}\right\}$ be a quasi boundary triple for $A^{*}$ with $A_{0}=\operatorname{ker} \Gamma_{0}$. Then the (operator-valued) functions $\gamma$ and $M$ defined by

$$
\gamma(\lambda):=\pi_{1}\left(\Gamma_{0} \uparrow \widehat{\mathcal{N}}_{\lambda, T}\right)^{-1} \quad \text { and } \quad M(\lambda):=\Gamma_{1}\left(\Gamma_{0}\left\lceil\widehat{\mathcal{N}}_{\lambda, T}\right)^{-1}, \quad \lambda \in \rho\left(A_{0}\right),\right.
$$

are called the $\gamma$-field and Weyl function corresponding to the quasi boundary triple $\left\{\mathcal{G}, \Gamma_{0}, \Gamma_{1}\right\}$.

Note that $\gamma(\lambda)$ is a mapping from $\mathcal{G}_{0}$ to $\mathcal{H}$, and $M(\lambda)$ is a mapping from $\mathcal{G}_{0}$ to $\mathcal{G}_{1} \subset \mathcal{G}$ for $\lambda \in \rho\left(A_{0}\right)$. These definitions coincide with the definition of the $\gamma$-field and Weyl function or Weyl family in the case where $\left\{\mathcal{G}, \Gamma_{0}, \Gamma_{1}\right\}$ is an ordinary boundary triple, generalized boundary triple or a boundary relation as in $[24,26,27]$. In the next proposition we collect some properties of the $\gamma$-field and the Weyl function of a quasi boundary triple, which are extensions of well-known properties of the $\gamma$-field and Weyl function of an ordinary boundary triple. The first six items were stated and proved in $[10$, Proposition 2.6].

Proposition 3.3. Let $A$ be a closed symmetric relation in $\mathcal{H}$ and let $\left\{\mathcal{G}, \Gamma_{0}, \Gamma_{1}\right\}$ be a quasi boundary triple for $A^{*}$ with $\gamma$-field $\gamma$ and Weyl function $M$. For $\lambda, \mu \in \rho\left(A_{0}\right)$ the following assertions hold.

(i) $\gamma(\lambda)$ is a densely defined bounded operator from $\mathcal{G}$ into $\mathcal{H}$ with domain $\operatorname{dom} \gamma(\lambda)=\mathcal{G}_{0}, \overline{\gamma(\lambda)} \in \mathcal{B}(\mathcal{G}, \mathcal{H})$, the function $\lambda \mapsto \gamma(\lambda) g$ is holomorphic on $\rho\left(A_{0}\right)$ for every $g \in \mathcal{G}_{0}$, and the relation

$$
\gamma(\lambda)=\left(I+(\lambda-\mu)\left(A_{0}-\lambda\right)^{-1}\right) \gamma(\mu)
$$

holds.

(ii) $\gamma(\bar{\lambda})^{*} \in \mathcal{B}(\mathcal{H}, \mathcal{G})$, $\operatorname{ran} \gamma(\bar{\lambda})^{*} \subset \mathcal{G}_{1}$ and for all $h \in \mathcal{H}$ we have

$$
\gamma(\bar{\lambda})^{*} h=\Gamma_{1}\left(\begin{array}{c}
\left(A_{0}-\lambda\right)^{-1} h \\
\left(I+\lambda\left(A_{0}-\lambda\right)^{-1}\right) h
\end{array}\right) .
$$

(iii) $M(\lambda)$ maps $\mathcal{G}_{0}$ into $\mathcal{G}_{1}$. If, in addition, $A_{1}:=\operatorname{ker} \Gamma_{1} \subset T$ is a self-adjoint relation in $\mathcal{H}$ and $\lambda \in \rho\left(A_{1}\right)$, then $M(\lambda)$ maps $\mathcal{G}_{0}$ onto $\mathcal{G}_{1}$.

(iv) $M(\lambda) \Gamma_{0} \hat{f}_{\lambda}=\Gamma_{1} \hat{f}_{\lambda}$ for all $\hat{f}_{\lambda} \in \widehat{\mathcal{N}}_{\lambda, T}$.

(v) $M(\lambda) \subset M(\bar{\lambda})^{*}$ and $M(\lambda)-M(\mu)^{*}=(\lambda-\bar{\mu}) \gamma(\mu)^{*} \gamma(\lambda)$. The function $\lambda \mapsto M(\lambda)$ is holomorphic in the sense that it can be written as the sum of the possibly unbounded operator $\operatorname{Re} M(\mu)$ and a bounded holomorphic operator function,

$$
\begin{aligned}
& M(\lambda)=\operatorname{Re} M(\mu) \\
& \quad+\gamma(\mu)^{*}\left((\lambda-\operatorname{Re} \mu)+(\lambda-\mu)(\lambda-\bar{\mu})\left(A_{0}-\lambda\right)^{-1}\right) \gamma(\mu) .
\end{aligned}
$$

(vi) $\operatorname{Im} M(\lambda)=\frac{1}{2 i}(M(\lambda)-M(\bar{\lambda}))$ is a densely defined bounded operator in $\mathcal{G}$. For $\lambda \in \mathbb{C}^{+}\left(\mathbb{C}^{-}\right)$the operator $\operatorname{Im} M(\lambda)$ is positive (negative, respectively). 
(vii) For $x \in \mathcal{G}_{0}$ the function $\lambda \mapsto M(\lambda) x$ is differentiable on $\rho\left(A_{0}\right)$ and

$$
\frac{d}{d \lambda} M(\lambda) x=\gamma(\bar{\lambda})^{*} \gamma(\lambda) x, \quad \lambda \in \rho\left(A_{0}\right) .
$$

(viii) If $M\left(\lambda_{0}\right)$ is bounded for some $\lambda_{0} \in \rho\left(A_{0}\right)$, then $M(\lambda)$ is bounded for all $\lambda \in \rho\left(A_{0}\right)$. In this case,

$$
\frac{1}{\operatorname{Im} \lambda} \operatorname{Im} \overline{M(\lambda)}>0, \quad \lambda \in \mathbb{C} \backslash \mathbb{R}
$$

and, in particular, $\operatorname{ker} \overline{M(\lambda)}=\{0\}$ for $\lambda \in \mathbb{C} \backslash \mathbb{R}$.

Proof. Items (i)-(vi) were proved in [10, Proposition 2.6]. To show (vii), let $x \in \mathcal{G}_{0}$ and $\lambda_{0}, \lambda \in \rho\left(A_{0}\right)$. It follows from (v) with $\mu=\overline{\lambda_{0}}$ that

$$
\frac{1}{\lambda-\lambda_{0}}\left(M(\lambda) x-M\left(\lambda_{0}\right) x\right)=\frac{1}{\lambda-\lambda_{0}}\left(M(\lambda) x-M\left(\overline{\lambda_{0}}\right)^{*} x\right)=\gamma\left(\overline{\lambda_{0}}\right)^{*} \gamma(\lambda) x .
$$

If we let $\lambda \rightarrow \lambda_{0}$, then the right-hand side converges, which shows that the derivative exists and that (3.4) is true for $\lambda$ replaced by $\lambda_{0}$. Item (viii) was shown in the case where $A^{*}$ is an operator in [11, Proposition 6.14 (vi)] The proof in the case of a relation is similar and hence omitted.

Remark 3.4. Note that the closure of the operator on the right-hand side of (3.4) is $\gamma(\bar{\lambda})^{*} \overline{\gamma(\lambda)}$, which is in $\mathcal{B}(\mathcal{G})$. Hence also $\frac{d}{d \lambda} M(\lambda)$ has a bounded, everywhere defined closure, which we denote by $\overline{M^{\prime}(\lambda)}$. With this notation we have the identity

$$
\overline{M^{\prime}(\lambda)}=\gamma(\bar{\lambda}) * \overline{\gamma(\lambda)}
$$

For the rest of this subsection we assume that $A$ is a closed symmetric relation in a separable Hilbert space $\mathcal{H}$. If $\left\{\mathcal{G}, \Gamma_{0}, \Gamma_{1}\right\}$ is a quasi boundary triple for $A^{*}$, then also the Hilbert space $\mathcal{G}$ is separable. The following proposition shows that, roughly speaking, the property of $\overline{\gamma(\lambda)}, \gamma(\lambda)^{*}$ and $\overline{M(\lambda)}$ belonging to some two-sided operator ideal is independent of $\lambda$.

Proposition 3.5. Let $A$ be a closed symmetric relation in a separable Hilbert space $\mathcal{H}$ and let $\left\{\mathcal{G}, \Gamma_{0}, \Gamma_{1}\right\}$ be a quasi boundary triple for $A^{*}$ with $A_{0}=\operatorname{ker} \Gamma_{0}$, $\gamma$-field $\gamma$ and Weyl function $M$. Moreover, let $\mathfrak{A}$ be a class of operator ideals. Then the following assertions are true.

(i) If $\overline{\gamma\left(\lambda_{0}\right)} \in \mathfrak{A}(\mathcal{G}, \mathcal{H})$ for some $\lambda_{0} \in \rho\left(A_{0}\right)$, then $\overline{\gamma(\lambda)} \in \mathfrak{A}(\mathcal{G}, \mathcal{H})$ for all $\lambda \in \rho\left(A_{0}\right)$.

(ii) If $\gamma\left(\lambda_{0}\right)^{*} \in \mathfrak{A}^{*}(\mathcal{H}, \mathcal{G})$ for some $\lambda_{0} \in \rho\left(A_{0}\right)$, then $\gamma(\lambda)^{*} \in \mathfrak{A}^{*}(\mathcal{H}, \mathcal{G})$ for all $\lambda \in \rho\left(A_{0}\right)$.

(iii) Assume that, in addition, $\mathfrak{A}$ is a class of symmetrically normed ideals and that $\overline{M\left(\lambda_{0}\right)} \in \mathfrak{A}(\mathcal{G})$ for some $\lambda_{0} \in \mathbb{C} \backslash \mathbb{R}$. Then $\overline{M(\lambda)} \in \mathfrak{A}(\mathcal{G})$ for all $\lambda \in \rho\left(A_{0}\right)$.

Proof. (i) It follows immediately from $I+\left(\lambda-\lambda_{0}\right)\left(A_{0}-\lambda\right)^{-1} \in \mathcal{B}(\mathcal{H})$ and Proposition 3.3 (i) that

$$
\overline{\gamma(\lambda)}=\left(I+\left(\lambda-\lambda_{0}\right)\left(A_{0}-\lambda\right)^{-1}\right) \overline{\gamma\left(\lambda_{0}\right)}
$$


holds for all $\lambda, \lambda_{0} \in \rho\left(A_{0}\right)$. The ideal property directly implies the assertion.

(ii) If $\gamma\left(\lambda_{0}\right)^{*} \in \mathfrak{A}(\mathcal{H}, \mathcal{G})$, then $\overline{\gamma\left(\lambda_{0}\right)}=\gamma\left(\lambda_{0}\right)^{* *} \in \mathfrak{A}^{*}(\mathcal{G}, \mathcal{H})$. By (i) this implies that $\overline{\gamma(\lambda)} \in \mathfrak{A}^{*}(\mathcal{G}, \mathcal{H})$ for all $\lambda \in \rho\left(A_{0}\right)$ and hence $\gamma(\lambda)^{*} \in \mathfrak{A}(\mathcal{H}, \mathcal{G})$ for all $\lambda \in \rho\left(A_{0}\right)$.

(iii) Assume that $\overline{M\left(\lambda_{0}\right)} \in \mathfrak{A}(\mathcal{G})$ for some $\lambda_{0} \in \mathbb{C} \backslash \mathbb{R}$. Then also Re $\overline{M\left(\lambda_{0}\right)}$ and $\operatorname{Im} \overline{M\left(\lambda_{0}\right)}$ belong to $\mathfrak{A}(\mathcal{G})$, and by Proposition $3.3(\mathrm{v})$ we have

$$
\frac{1}{\operatorname{Im} \lambda_{0}} \operatorname{Im} \overline{M\left(\lambda_{0}\right)}=\gamma\left(\lambda_{0}\right)^{*} \overline{\gamma\left(\lambda_{0}\right)} \in \mathfrak{A}(\mathcal{G}) .
$$

Since $\overline{\gamma\left(\lambda_{0}\right)} \in \mathcal{B}(\mathcal{G}, \mathcal{H})$ and $\gamma\left(\lambda_{0}\right)^{*}={\overline{\gamma\left(\lambda_{0}\right)}}^{*} \in \mathcal{B}(\mathcal{H}, \mathcal{G})$, we can use Lemma 2.4 to conclude that for every $\lambda \in \rho\left(A_{0}\right)$ also

$$
\gamma\left(\lambda_{0}\right)^{*}\left(\left(\lambda-\operatorname{Re} \lambda_{0}\right)+\left(\lambda-\lambda_{0}\right)\left(\lambda-\overline{\lambda_{0}}\right)\left(A_{0}-\lambda\right)^{-1}\right) \overline{\gamma\left(\lambda_{0}\right)} \in \mathfrak{A}(\mathcal{G}) .
$$

It follows from Proposition $3.3(\mathrm{v})$ that for $\lambda \in \rho\left(A_{0}\right)$ we have

$\overline{M(\lambda)}=\operatorname{Re} \overline{M\left(\lambda_{0}\right)}+\gamma\left(\lambda_{0}\right)^{*}\left(\left(\lambda-\operatorname{Re} \lambda_{0}\right)+\left(\lambda-\lambda_{0}\right)\left(\lambda-\overline{\lambda_{0}}\right)\left(A_{0}-\lambda\right)^{-1}\right) \overline{\gamma\left(\lambda_{0}\right)}$.

Therefore $\operatorname{Re} \overline{M\left(\lambda_{0}\right)} \in \mathfrak{A}(\mathcal{G})$ and (3.7) imply that $\overline{M(\lambda)} \in \mathfrak{A}(\mathcal{G})$ for all $\lambda \in$ $\rho\left(A_{0}\right)$.

Remark 3.6. Note that in Proposition 3.5 (iii) it is assumed that $\lambda_{0}$ is nonreal. However, it follows from the proof of Proposition 3.5 (iii) that the assumptions $\overline{M\left(\lambda_{1}\right)} \in \mathfrak{A}(\mathcal{G})$ and $\gamma\left(\lambda_{1}\right)^{*} \overline{\gamma\left(\lambda_{1}\right)} \in \mathfrak{A}(\mathcal{G})$ for some $\lambda_{1} \in \mathbb{R} \cap \rho\left(A_{0}\right)$ also yield $\overline{M(\lambda)} \in \mathfrak{A}(\mathcal{G})$ for all $\lambda \in \rho\left(A_{0}\right)$. On the other hand, the assumption $\overline{M\left(\lambda_{1}\right)} \in \mathfrak{A}(\mathcal{G})$ for some $\lambda_{1} \in \mathbb{R} \cap \rho\left(A_{0}\right)$ alone does not imply that $\overline{M(\lambda)} \in \mathfrak{A}(\mathcal{G})$ for all $\lambda \in \rho\left(A_{0}\right)$.

Proposition 3.7. Let $\mathfrak{A}$ be a class of operator ideals. Moreover, let $\gamma$ be the $\gamma$-field associated with some quasi boundary triple $\left\{\mathcal{G}, \Gamma_{0}, \Gamma_{1}\right\}$, let $\widetilde{\mathcal{G}}_{1}$ be a Hilbert space such that $\mathcal{G}_{1} \subset \widetilde{\mathcal{G}}_{1} \subset \mathcal{G}$ and the embedding $\iota_{\widetilde{\mathcal{G}}_{1} \rightarrow \mathcal{G}}$ belongs to $\mathfrak{A}\left(\widetilde{\mathcal{G}}_{1}, \mathcal{G}\right)$. Then

$$
\gamma(\lambda)^{*} \in \mathfrak{A}(\mathcal{H}, \mathcal{G})
$$

for all $\lambda \in \rho\left(A_{0}\right)$.

Proof. For every $\lambda \in \rho\left(A_{0}\right)$ we have $\gamma(\lambda)^{*} \in \mathcal{B}(\mathcal{H}, \mathcal{G})$ and $\operatorname{ran} \gamma(\lambda)^{*} \subset \mathcal{G}_{1}$ by Proposition 3.3 (ii). Hence $\gamma(\lambda)^{*}$ is closed as an operator from $\mathcal{H}$ to $\mathcal{G}$. Since $\iota_{\iota_{\mathcal{G}_{1} \rightarrow \mathcal{G}}}$ is bounded, $\gamma(\lambda)^{*}$ regarded as an operator from $\mathcal{H}$ into $\widetilde{\mathcal{G}}_{1}$ is also closed and hence bounded by the closed graph theorem, that is, $\gamma(\lambda)^{*} \in \mathcal{B}\left(\mathcal{H}, \widetilde{\mathcal{G}}_{1}\right)$. Hence, by the ideal property, (3.8) holds.

\subsection{Krein's formula and self-adjoint extensions}

The following theorem and corollary contain a variant of Krein's formula for the resolvents of canonical extensions parameterized with the help of quasi boundary triples, cf. (3.2) and (3.3). 
Theorem 3.8. Let $A$ be a closed symmetric relation in $\mathcal{H}$ and let $\left\{\mathcal{G}, \Gamma_{0}, \Gamma_{1}\right\}$ be a quasi boundary triple for $A^{*}$ with $A_{0}=\operatorname{ker} \Gamma_{0}, \gamma$-field $\gamma$ and Weyl function $M$. Further, let $\Theta$ be a relation in $\mathcal{G}$ and assume that $\lambda \in \rho\left(A_{0}\right)$ is not an eigenvalue of $A_{\Theta}$, or, equivalently, that $\operatorname{ker}(\Theta-M(\lambda))=\{0\}$. Then the following assertions are true:

(i) $g \in \operatorname{ran}\left(A_{\Theta}-\lambda\right)$ if and only if $\gamma(\bar{\lambda})^{*} g \in \operatorname{dom}(\Theta-M(\lambda))^{-1}$;

(ii) for all $g \in \operatorname{ran}\left(A_{\Theta}-\lambda\right)$ we have

$$
\left(A_{\Theta}-\lambda\right)^{-1} g=\left(A_{0}-\lambda\right)^{-1} g+\gamma(\lambda)(\Theta-M(\lambda))^{-1} \gamma(\bar{\lambda})^{*} g .
$$

Proof. First note that, by [10, Theorem 2.8(i)], the point $\lambda \in \rho\left(A_{0}\right)$ is not an eigenvalue of $A_{\Theta}$ if and only if $\operatorname{ker}(\Theta-M(\lambda))=\{0\}$. Fix some point $\lambda \in \rho\left(A_{0}\right)$ which is not an eigenvalue of $A_{\Theta}$. Then the inverses $\left(A_{\Theta}-\lambda\right)^{-1}$ and $(\Theta-M(\lambda))^{-1}$ are operators in $\mathcal{H}$ and $\mathcal{G}$, respectively.

The implication " $\Leftarrow$ " in (i) and item (ii) were shown in the proof of [10, Theorem 2.8 (ii)]. The converse implication in (i) was proved in [11, Theorem 6.16] for the case when $A^{*}$ is an operator. Therefore we only outline the proof for the case when $A^{*}$ is a relation. For given $g \in \operatorname{ran}\left(A_{\Theta}-\lambda\right)$ set

$$
\widehat{h}:=\left(\begin{array}{c}
\left(A_{\Theta}-\lambda\right)^{-1} g \\
g+\lambda\left(A_{\Theta}-\lambda\right)^{-1} g
\end{array}\right) .
$$

One can show in a similar way as in $[11$, Theorem 6.16$]$ that

$$
\left(\begin{array}{c}
\Gamma_{0} \widehat{h} \\
\gamma(\bar{\lambda})^{*} g
\end{array}\right)=\left(\begin{array}{c}
\Gamma_{0} \widehat{h} \\
\Gamma_{1} \widehat{h}-M(\lambda) \Gamma_{0} \widehat{h}
\end{array}\right) \in \Theta-M(\lambda),
$$

which implies that $\gamma(\bar{\lambda})^{*} g \in \operatorname{ran}(\Theta-M(\lambda))=\operatorname{dom}(\Theta-M(\lambda))^{-1}$.

If $\rho\left(A_{\Theta}\right) \cap \rho\left(A_{0}\right) \neq \varnothing$ or $\rho\left(\overline{A_{\Theta}}\right) \cap \rho\left(A_{0}\right) \neq \varnothing$, e.g. if $A_{\Theta}$ is self-adjoint or essentially self-adjoint, respectively, then, for $\lambda \in \rho\left(\overline{A_{\Theta}}\right) \cap \rho\left(A_{0}\right)$, relation (3.9) is valid on $\mathcal{H}$ or a dense subset of $\mathcal{H}$, respectively. This, together with the fact that $\gamma(\bar{\lambda})^{*}$ is an everywhere defined bounded operator and

$$
\gamma(\lambda)(\Theta-M(\lambda))^{-1} \gamma(\bar{\lambda})^{*} \subset \overline{\gamma(\lambda)(\Theta-M(\lambda))^{-1}} \gamma(\bar{\lambda})^{*},
$$

implies the following corollary.

Corollary 3.9. Let the assumptions be as in Theorem 3.8. Then the following assertions hold.

(i) If $\lambda \in \rho\left(A_{\Theta}\right) \cap \rho\left(A_{0}\right)$, then

$$
\left(A_{\Theta}-\lambda\right)^{-1}=\left(A_{0}-\lambda\right)^{-1}+\gamma(\lambda)(\Theta-M(\lambda))^{-1} \gamma(\bar{\lambda})^{*} .
$$

(ii) If $\lambda \in \rho\left(\overline{A_{\Theta}}\right) \cap \rho\left(A_{0}\right)$, then

$$
\left(\overline{A_{\Theta}}-\lambda\right)^{-1}=\left(A_{0}-\lambda\right)^{-1}+\overline{\gamma(\lambda)(\Theta-M(\lambda))^{-1}} \gamma(\bar{\lambda})^{*} .
$$


In particular, if $A_{\Theta}$ is self-adjoint or essentially self-adjoint, then Krein's formula (3.10) or (3.11), respectively, holds at least for all non-real $\lambda$.

With the help of Krein's formula and the next lemma we obtain a sufficient condition for self-adjointness of extensions $A_{\Theta}$ in Theorem 3.11 below.

Lemma 3.10. Let $\left\{\mathcal{G}, \Gamma_{0}, \Gamma_{1}\right\}$ be a quasi boundary triple with associated Weyl function $M$. Assume that $\overline{M\left(\lambda_{0}\right)} \in \mathfrak{S}_{\infty}(\mathcal{G})$ for some $\lambda_{0} \in \mathbb{C} \backslash \mathbb{R}$ and let $\Theta$ be a self-adjoint relation in $\mathcal{G}$ such that $0 \notin \sigma_{\mathrm{ess}}(\Theta)$. Then

$$
(\Theta-\overline{M(\lambda)})^{-1} \in \mathcal{B}(\mathcal{G})
$$

for all $\lambda \in \mathbb{C} \backslash \mathbb{R}$.

Proof. According to Proposition 3.5 (iii) the operator $\overline{M(\lambda)}$ is compact for all $\lambda \in \mathbb{C} \backslash \mathbb{R}$ because $\mathfrak{S}_{\infty}(\mathcal{G})$ is a symmetrically normed ideal. Without loss of generality let $\lambda \in \mathbb{C}^{+}$in the following. We can decompose the self-adjoint relation $\Theta$ into its self-adjoint operator part and the purely multi-valued part: $\Theta=\Theta_{\text {op }} \oplus \Theta_{\infty}$ with a corresponding decomposition of the space $\mathcal{G}=\mathcal{G}_{\text {op }} \oplus \mathcal{G}_{\infty}$, cf. Section 2.1. Denote by $P_{\text {op }}$ the orthogonal projection in $\mathcal{G}$ onto $\mathcal{G}_{\text {op }}$. Since $0 \notin \sigma_{\text {ess }}\left(\Theta_{\text {op }}\right)$ and $\overline{M(\lambda)}$ is compact, the operator $\Theta_{\text {op }}-\left.P_{\text {op }} \overline{M(\lambda)}\right|_{\mathcal{G}_{\text {op }}}$ is a Fredholm operator in $\mathcal{G}_{\text {op }}$ with index 0 . For $x \in \operatorname{dom} \Theta_{\text {op }}, x \neq 0$, we have

$$
\begin{aligned}
\operatorname{Im}\left(\left(\Theta_{\mathrm{op}}-\left.P_{\mathrm{op}} \overline{M(\lambda)}\right|_{\mathcal{G}_{\mathrm{op}}}\right) x, x\right)_{\mathcal{G}_{\mathrm{op}}} & =-\operatorname{Im}(\overline{M(\lambda)} x, x) \\
& =-((\operatorname{Im} \overline{M(\lambda)}) x, x)<0
\end{aligned}
$$

by Proposition 3.3 (viii); hence $\Theta_{\text {op }}-\left.P_{\text {op }} \overline{M(\lambda)}\right|_{\mathcal{G}_{\text {op }}}$ has a trivial kernel. Since its index is zero, it is also surjective. Because of the closedness, its inverse is a bounded and everywhere defined operator in $\mathcal{G}_{\text {op }}$. By $[49$, p. 137] we have

$$
(\Theta-\overline{M(\lambda)})^{-1}=\left(\Theta_{\mathrm{op}}-\left.P_{\mathrm{op}} \overline{M(\lambda)}\right|_{\mathcal{G}_{\mathrm{op}}}\right)^{-1} P_{\mathrm{op}}
$$

and hence $(\Theta-\overline{M(\lambda)})^{-1} \in \mathcal{B}(\mathcal{G})$.

In the assumptions of the next theorem, which is one of the main results of the paper, we make use of the notation

$$
\Theta^{-1}(X):=\left\{x \in \mathcal{G}: \exists y \in X \text { so that }\left(\begin{array}{l}
x \\
y
\end{array}\right) \in \Theta\right\}
$$

for a linear relation $\Theta$ in $\mathcal{G}$ and a subspace $X \subset \mathcal{G}$. This theorem gives a sufficient condition for $A_{\Theta}$ being self-adjoint.

Theorem 3.11. Let $A$ be a closed symmetric relation in $\mathcal{H}$ and let $\left\{\mathcal{G}, \Gamma_{0}, \Gamma_{1}\right\}$ be a quasi boundary triple for $A^{*}$ with $A_{i}=\operatorname{ker} \Gamma_{i}, i=0,1$, and Weyl function $M$. Assume that $A_{1}$ is self-adjoint and that $\overline{M\left(\lambda_{0}\right)} \in \mathfrak{S}_{\infty}(\mathcal{G})$ for some $\lambda_{0} \in$ $\mathbb{C} \backslash \mathbb{R}$. If $\Theta$ is a self-adjoint relation in $\mathcal{G}$ such that

$$
0 \notin \sigma_{\text {ess }}(\Theta) \quad \text { and } \quad \Theta^{-1}\left(\operatorname{ran} \overline{M\left(\lambda_{ \pm}\right)}\right) \subset \mathcal{G}_{0}
$$

hold for some $\lambda_{+} \in \mathbb{C}^{+}$and some $\lambda_{-} \in \mathbb{C}^{-}$, then $A_{\Theta}=\{\hat{f} \in T: \Gamma \hat{f} \in \Theta\}$ is self-adjoint in $\mathcal{H}$. In particular, the second condition in (3.12) is satisfied if $\operatorname{dom} \Theta \subset \mathcal{G}_{0}$. 
Proof. Note first that $\Theta=\Theta^{*}$ implies that $A_{\Theta}$ is a symmetric relation in $\mathcal{H}$ and hence the eigenvalues of $A_{\Theta}$ are real. Therefore it remains to check that $\operatorname{ran}\left(A_{\Theta}-\lambda_{ \pm}\right)=\mathcal{H}$ holds for some (and hence for all) points $\lambda_{ \pm} \in \mathbb{C}^{ \pm}$. Since $\operatorname{ran} \gamma\left(\bar{\lambda}_{ \pm}\right)^{*} \subset \mathcal{G}_{1}$ by Proposition 3.3 (ii), we find from Theorem 3.8 (i) that it is sufficient to verify the inclusion

$$
\mathcal{G}_{1} \subset \operatorname{dom}\left(\Theta-M\left(\lambda_{ \pm}\right)\right)^{-1}=\operatorname{ran}\left(\Theta-M\left(\lambda_{ \pm}\right)\right) .
$$

Let $y \in \mathcal{G}_{1}$ and let $\lambda_{+} \in \mathbb{C}^{+}$be such that the second relation in (3.12) holds. For $\lambda_{-} \in \mathbb{C}^{-}$the same reasoning applies. With $x:=\left(\Theta-\overline{M\left(\lambda_{+}\right)}\right)^{-1} y$, which is well defined by Lemma 3.10, we have

$$
\left(\begin{array}{c}
x \\
y+\overline{M\left(\lambda_{+}\right)} x
\end{array}\right) \in \Theta .
$$

Since $A_{1}$ is self-adjoint, we have $\operatorname{ran} M\left(\lambda_{+}\right)=\mathcal{G}_{1}$ by Proposition 3.3 (iii) and hence

$$
y+\overline{M\left(\lambda_{+}\right)} x \in \mathcal{G}_{1}+\operatorname{ran} \overline{M\left(\lambda_{+}\right)}=\operatorname{ran} \overline{M\left(\lambda_{+}\right)} .
$$

It follows from the second assumption in (3.12) that $x \in \mathcal{G}_{0}=\operatorname{dom} M\left(\lambda_{+}\right)$. Therefore $\left(\begin{array}{l}x \\ y\end{array}\right) \in \Theta-M\left(\lambda_{+}\right)$, which shows that $y \in \operatorname{ran}\left(\Theta-M\left(\lambda_{+}\right)\right)$.

Remark 3.12. If $\Theta$ is a self-adjoint relation with $0 \notin \sigma_{\mathrm{ess}}(\Theta)$, then its kernel is finite-dimensional. If $\operatorname{ker} \Theta=\{0\}$, then $B:=\Theta^{-1}$ is a bounded, self-adjoint operator in $\mathcal{G}$. In this case, the second condition in (3.12) becomes

$$
B\left(\operatorname{ran} \overline{M\left(\lambda_{ \pm}\right)}\right) \subset \mathcal{G}_{0}
$$

and the relation $A_{\Theta}$ can be written as $A_{\Theta}=\operatorname{ker}\left(B \Gamma_{1}-\Gamma_{0}\right)$. If $\operatorname{ker} \Theta \neq\{0\}$, then one can write the abstract boundary condition $\Gamma \hat{f} \in \Theta, \hat{f} \in T \subset A^{*}$, with the finite rank projection $P$ onto $\operatorname{ker} \Theta$ and the bounded operator

$$
B=\left(\Theta \cap\left((\operatorname{ker} \Theta)^{\perp} \times(\operatorname{ker} \Theta)^{\perp}\right)\right)^{-1} \in \mathcal{B}\left((\operatorname{ker} \Theta)^{\perp}\right)
$$

in the form

$$
P \Gamma_{1} \hat{f}=0 \quad \text { and } \quad(1-P) \Gamma_{0} \hat{f}=B(1-P) \Gamma_{1} \hat{f}, \quad \hat{f} \in \operatorname{dom} \Gamma=T .
$$

\subsection{Resolvent differences in operator ideals}

Let $A$ be a closed symmetric relation in a separable Hilbert space $\mathcal{H}$, let $\left\{\mathcal{G}, \Gamma_{0}, \Gamma_{1}\right\}$ be a quasi boundary triple for $A^{*}$, and let $\mathfrak{A}$ be a class of operator ideals. With the help of Krein's formula we find sufficient conditions on the parameter $\Theta$, the $\gamma$-field $\gamma$ and the Weyl function $M$ such that the difference of the resolvents of the self-adjoint relations $A_{\Theta}$ and $A_{0}$ belongs to some appropriate ideal, e.g. $\mathfrak{A}(\mathcal{H})$ or $\left(\mathfrak{A} \cdot \mathfrak{A}^{*}\right)(\mathcal{H})$. These abstract results will turn out to be particularly useful in Section 4 when we investigate Schattenvon Neumann type properties of resolvent differences of self-adjoint elliptic differential operators.

The first theorem of this subsection is one of the main results of the paper. Here we consider the resolvent difference of $A_{\Theta}$ and $A_{0}$ under some assumptions on $M(\lambda), \gamma(\lambda)^{*}$ and $\Theta$. 
Theorem 3.13. Let $A$ be a closed symmetric relation in $\mathcal{H}$ and let $\left\{\mathcal{G}, \Gamma_{0}, \Gamma_{1}\right\}$ be a quasi boundary triple for $A^{*}$ with $A_{0}=\operatorname{ker} \Gamma_{0}, \gamma$-field $\gamma$ and Weyl function $M$. Let $\mathfrak{A}$ be a class of operator ideals and let $\Theta$ be a self-adjoint relation in $\mathcal{G}$ such that the following conditions hold:

(i) $\overline{M\left(\lambda_{0}\right)} \in \mathfrak{S}_{\infty}(\mathcal{G})$ for some $\lambda_{0} \in \mathbb{C} \backslash \mathbb{R}$;

(ii) $\gamma\left(\lambda_{1}\right)^{*} \in \mathfrak{A}^{*}(\mathcal{H}, \mathcal{G})$ for some $\lambda_{1} \in \rho\left(A_{0}\right)$;

(iii) $0 \notin \sigma_{\mathrm{ess}}(\Theta)$ and $A_{\Theta}=A_{\Theta}^{*}$.

Then

$$
\left(A_{\Theta}-\lambda\right)^{-1}-\left(A_{0}-\lambda\right)^{-1} \in\left(\mathfrak{A} \cdot \mathfrak{A}^{*}\right)(\mathcal{H})
$$

for all $\lambda \in \rho\left(A_{\Theta}\right) \cap \rho\left(A_{0}\right)$.

Proof. Note that the assumptions (i) and (ii) together with Proposition 3.5 imply that $\overline{M(\lambda)} \in \mathfrak{S}_{\infty}(\mathcal{G}), \gamma(\lambda)^{*} \in \mathfrak{A}^{*}(\mathcal{H}, \mathcal{G})$ and $\gamma(\lambda)^{* *}=\overline{\gamma(\lambda)} \in \mathfrak{A}(\mathcal{G}, \mathcal{H})$ for all $\lambda \in \rho\left(A_{0}\right)$. Corollary 3.9 (i) yields that the resolvent difference of the self-adjoint relations $A_{\Theta}$ and $A_{0}$ has the form

$$
\begin{aligned}
\left(A_{\Theta}-\lambda\right)^{-1}-\left(A_{0}-\lambda\right)^{-1} & =\gamma(\lambda)(\Theta-M(\lambda))^{-1} \gamma(\bar{\lambda})^{*} \\
& =\overline{\gamma(\lambda)}(\Theta-\overline{M(\lambda)})^{-1} \gamma(\bar{\lambda})^{*}
\end{aligned}
$$

for all $\lambda \in \rho\left(A_{\Theta}\right) \cap \rho\left(A_{0}\right)$. Furthermore, since the operator $\overline{M\left(\lambda_{0}\right)}$ is compact, we have $(\Theta-\overline{M(\lambda)})^{-1} \in \mathcal{B}(\mathcal{G})$ for all $\lambda \in \mathbb{C} \backslash \mathbb{R}$ by Lemma 3.10. Therefore, if $\lambda \in \mathbb{C} \backslash \mathbb{R}$, then

$$
(\Theta-\overline{M(\lambda)})^{-1} \gamma(\bar{\lambda})^{*} \in \mathfrak{A}^{*}(\mathcal{H}, \mathcal{G}) \text { and } \overline{\gamma(\lambda)} \in \mathfrak{A}(\mathcal{G}, \mathcal{H}),
$$

and hence (3.13) follows. Lemma 2.2 implies that (3.13) holds also for all $\lambda$ in the (possibly larger) set $\rho\left(A_{\Theta}\right) \cap \rho\left(A_{0}\right)$.

Note that Theorem 3.11 provides a sufficient condition for the second assumption in (iii) of Theorem 3.13 .

Remark 3.14. As a corollary one immediately obtains the same result for the resolvent difference

$$
\left(A_{\Theta_{1}}-\lambda\right)^{-1}-\left(A_{\Theta_{2}}-\lambda\right)^{-1}
$$

of $A_{\Theta_{1}}, A_{\Theta_{2}}$, where $\Theta_{1}$ and $\Theta_{2}$ both satisfy the assumptions in Theorem 3.13 . In Theorem 3.20 we improve this under the additional assumption that $\Theta_{1}-$ $\Theta_{2}$ is in some class of operator ideals.

Remark 3.15. If $\mathfrak{A}$ is equal to $\mathfrak{S}_{p}, \mathfrak{S}_{p, \infty}$ or $\mathfrak{S}_{p, \infty}^{(0)}$, then the resolvent difference in $(3.13)$ is in $\mathfrak{S}_{p / 2}(\mathcal{H}), \mathfrak{S}_{p / 2, \infty},(\mathcal{H})$ or $\mathfrak{S}_{p / 2, \infty}^{(0)}(\mathcal{H})$, respectively. This follows from Lemma 2.3 .

Krein's formula can be used to prove a trace formula if the resolvent difference is a trace class operator.

Corollary 3.16. Let $A$ be a closed symmetric relation in a separable Hilbert space $\mathcal{H}$ and let $\left\{\mathcal{G}, \Gamma_{0}, \Gamma_{1}\right\}$ be a quasi boundary triple for $A^{*}$ with $A_{0}=\operatorname{ker} \Gamma_{0}$, $\gamma$-field $\gamma$ and Weyl function $M$. Further, let $\Theta$ be a self-adjoint relation in $\mathcal{G}$ such that the following conditions hold: 
(i) $\overline{M\left(\lambda_{0}\right)} \in \mathfrak{S}_{\infty}(\mathcal{G})$ for some $\lambda_{0} \in \mathbb{C} \backslash \mathbb{R}$;

(ii) $\gamma\left(\lambda_{1}\right)^{*} \in \mathfrak{S}_{2}(\mathcal{H}, \mathcal{G})$ for some $\lambda_{1} \in \rho\left(A_{0}\right)$;

(iii) $0 \notin \sigma_{\text {ess }}(\Theta)$ and $A_{\Theta}=A_{\Theta}^{*}$.

Then

$$
\left(A_{\Theta}-\lambda\right)^{-1}-\left(A_{0}-\lambda\right)^{-1} \in \mathfrak{S}_{1}(\mathcal{H})
$$

and

$$
\operatorname{tr}\left(\left(A_{\Theta}-\lambda\right)^{-1}-\left(A_{0}-\lambda\right)^{-1}\right)=\operatorname{tr}\left(\overline{M^{\prime}(\lambda)}(\Theta-\overline{M(\lambda)})^{-1}\right)
$$

for $\lambda \in \rho\left(A_{\Theta}\right) \cap \rho\left(A_{0}\right)$, where $\overline{M^{\prime}(\lambda)}$ is defined as in Remark 3.4.

Proof. The first assertion is clear from Theorem 3.13 and Remark 3.15. Hence we can apply the trace to both sides of (3.14). Using (3.6) and the relation $\operatorname{tr}(A B)=\operatorname{tr}(B A)$ (see, e.g. [36, Theorem III.8.2]) we obtain

$$
\begin{aligned}
& \operatorname{tr}\left(\left(A_{\Theta}-\lambda\right)^{-1}-\left(A_{0}-\lambda\right)^{-1}\right)=\operatorname{tr}\left(\overline{\gamma(\lambda)}(\Theta-\overline{M(\lambda)})^{-1} \gamma(\bar{\lambda})^{*}\right) \\
& =\operatorname{tr}\left(\gamma(\bar{\lambda})^{*} \overline{\gamma(\lambda)}(\Theta-\overline{M(\lambda)})^{-1}\right)=\operatorname{tr}\left(\overline{M^{\prime}(\lambda)}(\Theta-\overline{M(\lambda)})^{-1}\right)
\end{aligned}
$$

note that also the operator $\overline{M^{\prime}(\lambda)}(\Theta-\overline{M(\lambda)})^{-1}$ in $(3.15)$ is a trace class operator.

In the following theorem the assumptions $\overline{M\left(\lambda_{0}\right)} \in \mathfrak{S}_{\infty}(\mathcal{G}), 0 \notin \sigma_{\text {ess }}(\Theta)$ are replaced by a weaker assumption on $\Theta-M(\lambda)$; the conclusion is also weaker than the one in Theorem 3.13.

Theorem 3.17. Let $A$ be a closed symmetric relation in $\mathcal{H}$ and let $\left\{\mathcal{G}, \Gamma_{0}, \Gamma_{1}\right\}$ be a quasi boundary triple for $A^{*}$ with $A_{0}=\operatorname{ker} \Gamma_{0}, \gamma$-field $\gamma$ and Weyl function $M$. Let $\mathfrak{A}$ be a class of operator ideals and let $\Theta$ be a symmetric relation in $\mathcal{G}$ such that the following conditions hold:

(i) $\overline{\Theta-M\left(\lambda_{0}\right)}$ is injective for some $\lambda_{0} \in \mathbb{C} \backslash \mathbb{R}$;

(ii) $\gamma\left(\lambda_{1}\right)^{*} \in \mathfrak{A}^{*}(\mathcal{H}, \mathcal{G})$ for some $\lambda_{1} \in \rho\left(A_{0}\right)$;

(iii) $A_{\Theta}=A_{\Theta}^{*}$.

Then

$$
\left(A_{\Theta}-\lambda\right)^{-1}-\left(A_{0}-\lambda\right)^{-1} \in \mathfrak{A}(\mathcal{H})
$$

for all $\lambda \in \rho\left(A_{\Theta}\right) \cap \rho\left(A_{0}\right)$.

Proof. According to Corollary 3.9 (i) we can write the resolvent difference at the point $\lambda_{0}$ as

$$
\left(A_{\Theta}-\lambda_{0}\right)^{-1}-\left(A_{0}-\lambda_{0}\right)^{-1}=\overline{\gamma\left(\lambda_{0}\right)}\left(\Theta-M\left(\lambda_{0}\right)\right)^{-1} \gamma\left(\bar{\lambda}_{0}\right)^{*} .
$$

In particular, it follows that both products on the right-hand side are well defined, and hence

$$
\left(\Theta-M\left(\lambda_{0}\right)\right)^{-1} \gamma\left(\bar{\lambda}_{0}\right)^{*}
$$

is everywhere defined. Since the relation $\overline{\Theta-M\left(\lambda_{0}\right)}$ is injective, it follows that $\left(\Theta-M\left(\lambda_{0}\right)\right)^{-1}$ is a closable operator. Therefore, because $\gamma\left(\bar{\lambda}_{0}\right)^{*}$ is a bounded operator, the product in (3.17) is a closable, everywhere defined 
operator and hence in $\mathcal{B}(\mathcal{H}, \mathcal{G})$. Moreover, since $\gamma\left(\lambda_{1}\right)^{*} \in \mathfrak{A}^{*}(\mathcal{H}, \mathcal{G})$, it follows from Proposition 3.5 that $\overline{\gamma(\lambda)}$ belongs to $\mathfrak{A}(\mathcal{G}, \mathcal{H})$ for all $\lambda \in \rho\left(A_{0}\right)$. Hence the difference of the resolvents in $(3.16)$ is in $\mathfrak{A}(\mathcal{H})$ for $\lambda=\lambda_{0}$. Then Lemma 2.2 implies (3.16) for all $\lambda \in \rho\left(A_{\Theta}\right) \cap \rho\left(A_{0}\right)$.

In the case $\Theta=0$ the above theorem, together with Proposition 3.3 (viii), implies the next corollary.

Corollary 3.18. Let $A,\left\{\mathcal{G}, \Gamma_{0}, \Gamma_{1}\right\}, \gamma, M$ and $\mathfrak{A}$ be as in Theorem 3.17. Assume that $A_{1}=\operatorname{ker} \Gamma_{1}$ is self-adjoint, that $M\left(\lambda_{0}\right)$ is bounded for some $\lambda_{0} \in \rho\left(A_{0}\right)$ and that $\gamma\left(\lambda_{1}\right)^{*} \in \mathfrak{A}^{*}(\mathcal{H}, \mathcal{G})$ for some $\lambda_{1} \in \rho\left(A_{0}\right)$. Then

$$
\left(A_{1}-\lambda\right)^{-1}-\left(A_{0}-\lambda\right)^{-1} \in \mathfrak{A}(\mathcal{H})
$$

for all $\lambda \in \rho\left(A_{1}\right) \cap \rho\left(A_{0}\right)$

Corollary 3.18 can be generalized as follows.

Theorem 3.19. Let $A$ be a closed symmetric relation in $\mathcal{H}$ and let $\left\{\mathcal{G}, \Gamma_{0}, \Gamma_{1}\right\}$ be a quasi boundary triple for $A^{*}$ with $A_{0}=\operatorname{ker} \Gamma_{0}, \gamma$-field $\gamma$ and Weyl function $M$. Furthermore, let $\mathfrak{A}, \mathfrak{B}$ be classes of operator ideals and assume that the following conditions hold:

(i) $A_{1}=\operatorname{ker} \Gamma_{1}$ is self-adjoint;

(ii) $M\left(\lambda_{0}\right)$ is bounded for some $\lambda_{0} \in \rho\left(A_{0}\right)$;

(iii) $\gamma\left(\lambda_{1}\right)^{*} \in \mathfrak{A}^{*}(\mathcal{H}, \mathcal{G})$ for some $\lambda_{1} \in \rho\left(A_{0}\right)$;

(iv) there exists a Hilbert space $\widetilde{\mathcal{G}}_{0}$ such that $\mathcal{G}_{0} \subset \widetilde{\mathcal{G}}_{0} \subset \mathcal{G}$ and the embedding $\iota_{\widetilde{\mathcal{G}}_{0} \rightarrow \mathcal{G}}$ belongs to $\mathfrak{B}\left(\widetilde{\mathcal{G}}_{0}, \mathcal{G}\right)$.

Then

$$
\left(A_{1}-\lambda\right)^{-1}-\left(A_{0}-\lambda\right)^{-1} \in(\mathfrak{A} \cdot \mathfrak{B})(\mathcal{H})
$$

for all $\lambda \in \rho\left(A_{1}\right) \cap \rho\left(A_{0}\right)$.

Proof. Since $M\left(\lambda_{0}\right)$ is bounded, Proposition 3.3 (viii) implies that $\operatorname{ker} \overline{M(\lambda)}=$ $\{0\}$ for every $\lambda \in \mathbb{C} \backslash \mathbb{R}$ and hence $M(\lambda)^{-1} \gamma(\bar{\lambda})^{*}$ is closable from $\mathcal{H}$ into $\mathcal{G}$ with values in $\mathcal{G}_{0}=\operatorname{dom} M(\lambda)$. The boundedness of the embedding $\iota_{\widetilde{\mathcal{G}}_{0} \rightarrow \mathcal{G}}$ implies that $M(\lambda)^{-1} \gamma(\bar{\lambda})^{*}$ regarded as an operator from $\mathcal{H}$ into $\widetilde{\mathcal{G}}_{0}$ is also closable. Furthermore, this operator is everywhere defined and hence we have

$$
M(\lambda)^{-1} \gamma(\bar{\lambda})^{*} \in \mathcal{B}\left(\mathcal{H}, \widetilde{\mathcal{G}}_{0}\right) \quad \text { and } \quad \iota_{\widetilde{\mathcal{G}}_{0} \rightarrow \mathcal{G}} M(\lambda)^{-1} \gamma(\bar{\lambda})^{*} \in \mathfrak{B}(\mathcal{H}, \mathcal{G})
$$

for all $\lambda \in \mathbb{C} \backslash \mathbb{R}$ by assumption (iv). Assumption (iii) implies $\overline{\gamma(\lambda)} \in \mathfrak{A}(\mathcal{G}, \mathcal{H})$ for all $\lambda \in \rho\left(A_{0}\right)$; cf. Proposition 3.5 (i). By the self-adjointness of $A_{1}$ and by Corollary 3.9 we have

$$
\left(A_{1}-\lambda\right)^{-1}-\left(A_{0}-\lambda\right)^{-1}=-\overline{\gamma(\lambda)} \iota_{\widetilde{\mathcal{G}}_{0} \rightarrow \mathcal{G}} M(\lambda)^{-1} \gamma(\bar{\lambda})^{*},
$$

which is in $(\mathfrak{A} \cdot \mathfrak{B})(\mathcal{H})$ for all $\lambda \in \mathbb{C} \backslash \mathbb{R}$. An application of Lemma 2.2 shows that (3.18) holds also for all $\lambda \in \rho\left(A_{1}\right) \cap \rho\left(A_{0}\right)$. 
In the next theorem the difference of the resolvents of two self-adjoint extensions $A_{\Theta_{1}}$ and $A_{\Theta_{2}}$ is considered under additional assumptions on $\Theta_{1}-$ $\Theta_{2}$; cf. [26, Theorem 2 and Corollary 4] for the case when $\left\{\mathcal{G}, \Gamma_{0}, \Gamma_{1}\right\}$ is an ordinary boundary triple.

Theorem 3.20. Let $A$ be a closed symmetric relation in $\mathcal{H}$ and let $\left\{\mathcal{G}, \Gamma_{0}, \Gamma_{1}\right\}$ be a quasi boundary triple for $A^{*}$ with $A_{0}=\operatorname{ker} \Gamma_{0}, \gamma$-field $\gamma$ and Weyl function $M$. Let $\mathfrak{B}$ be a class of operator ideals, let $\Theta_{1}$ and $\Theta_{2}$ be two selfadjoint bounded operators in $\mathcal{G}$ and assume that the following conditions hold:

(i) $\overline{M\left(\lambda_{0}\right)} \in \mathfrak{S}_{\infty}(\mathcal{G})$ for some $\lambda_{0} \in \rho\left(A_{0}\right)$;

(ii) $0 \notin \sigma_{\mathrm{ess}}\left(\Theta_{i}\right)$ and $A_{\Theta_{i}}=A_{\Theta_{i}}^{*}$ for $i=1,2$;

(iii) $\Theta_{1}-\Theta_{2} \in \mathfrak{B}(\mathcal{G})$.

Then

$$
\left(A_{\Theta_{1}}-\lambda\right)^{-1}-\left(A_{\Theta_{2}}-\lambda\right)^{-1} \in \mathfrak{B}(\mathcal{H})
$$

holds for all $\lambda \in \rho\left(A_{\Theta_{1}}\right) \cap \rho\left(A_{\Theta_{2}}\right)$. If, in addition, $\mathfrak{A}$ is another class of operator ideals and $\gamma\left(\lambda_{1}\right)^{*} \in \mathfrak{A}^{*}(\mathcal{H}, \mathcal{G})$ for some $\lambda_{1} \in \rho\left(A_{0}\right)$, then

$$
\left(A_{\Theta_{1}}-\lambda\right)^{-1}-\left(A_{\Theta_{2}}-\lambda\right)^{-1} \in\left(\mathfrak{A} \cdot \mathfrak{B} \cdot \mathfrak{A}^{*}\right)(\mathcal{H})
$$

for all $\lambda \in \rho\left(A_{\Theta_{1}}\right) \cap \rho\left(A_{\Theta_{2}}\right)$.

Proof. Since, by Lemma 3.10, the operators $\Theta_{i}$ and $\left(\Theta_{i}-\overline{M(\lambda)}\right)^{-1}$ are bounded for all $\lambda \in \mathbb{C} \backslash \mathbb{R}$, the difference of (3.10) for $\Theta=\Theta_{1}$ and $\Theta=\Theta_{2}$ can be rewritten as follows

$$
\begin{aligned}
\left(A_{\Theta_{1}}\right. & -\lambda)^{-1}-\left(A_{\Theta_{2}}-\lambda\right)^{-1} \\
& =\overline{\gamma(\lambda)}\left(\Theta_{1}-\overline{M(\lambda)}\right)^{-1}\left(\Theta_{2}-\Theta_{1}\right)\left(\Theta_{2}-\overline{M(\lambda)}\right)^{-1} \gamma(\bar{\lambda})^{*} .
\end{aligned}
$$

All five factors on the right-hand side are bounded, the middle factor is in $\mathfrak{B}(\mathcal{G})$; hence the product is in $\mathfrak{B}(\mathcal{H})$ for $\lambda \in \mathbb{C} \backslash \mathbb{R}$, and Lemma 2.2 implies that this is true for all $\lambda \in \rho\left(A_{\Theta_{1}}\right) \cap \rho\left(A_{\Theta_{2}}\right)$. If, in addition, $\gamma\left(\lambda_{1}\right)^{*} \in \mathfrak{A}^{*}(\mathcal{H}, \mathcal{G})$, then $\gamma(\bar{\lambda})^{*} \in \mathfrak{A}^{*}(\mathcal{H}, \mathcal{G})$ and $\overline{\gamma(\lambda)} \in \mathfrak{A}(\mathcal{G}, \mathcal{H})$ for all $\lambda \in \rho\left(A_{0}\right)$ by Proposition 3.5 (ii) and hence the second assertion holds for all $\lambda \in \rho\left(A_{\Theta_{1}}\right) \cap$ $\rho\left(A_{\Theta_{2}}\right) \cap \rho\left(A_{0}\right)$. It remains to use Lemma 2.2 to conclude the assertion for all $\lambda \in \rho\left(A_{\Theta_{1}}\right) \cap \rho\left(A_{\Theta_{2}}\right)$.

\section{Self-adjoint elliptic operators and spectral estimates for resolvent differences}

In this section we study elliptic operators on domains in $\mathbb{R}^{n}$ with smooth compact boundary, i.e. either on bounded domains or on exterior domains. In the first subsection we construct a quasi boundary triple where functions in the domain of $T$ are in $H^{2}$ in a neighbourhood of the boundary and prove sufficient conditions for self-adjoint realizations. We shall sometimes speak of an $H^{2}$ framework here although for exterior domains $T$ is defined on a larger space, see Definition 4.1. In Subsection 4.2 we apply the abstract results from Section 3.3 to elliptic operators and obtain estimates for singular values 
of resolvent differences of realizations with different boundary conditions. In Section 4.3 self-adjoint elliptic operators on $\mathbb{R}^{n}$ with $\delta$-interactions on smooth hypersurfaces are constructed with the help of quasi boundary triples and interface conditions on the hypersurface. The abstract results from Section 3.3 imply spectral estimates for the resolvent differences of the elliptic operators with $\delta$-interactions and the unperturbed elliptic operator on $\mathbb{R}^{n}$; cf. [13] for similar considerations for Schrödinger operators.

\subsection{Quasi boundary triples and Weyl functions for second order elliptic differential expressions}

Let $\Omega \subset \mathbb{R}^{n}$ be a bounded or unbounded domain with compact $C^{\infty}$-boundary $\partial \Omega$. We consider a formally symmetric second order differential expression

$$
(\mathcal{L} f)(x):=-\sum_{j, k=1}^{n}\left(\frac{\partial}{\partial x_{j}}\left(a_{j k} \frac{\partial f}{\partial x_{k}}\right)\right)(x)+a(x) f(x), \quad x \in \Omega,
$$

with bounded, infinitely differentiable, real-valued coefficients $a_{j k} \in C^{\infty}(\bar{\Omega})$ satisfying $a_{j k}(x)=a_{k j}(x)$ for all $x \in \bar{\Omega}$ and $j, k=1, \ldots, n$ and a real-valued function $a \in L^{\infty}(\Omega)$. Furthermore, $\mathcal{L}$ is assumed to be uniformly elliptic, i.e. the condition

$$
\sum_{j, k=1}^{n} a_{j k}(x) \xi_{j} \xi_{k} \geq C \sum_{k=1}^{n} \xi_{k}^{2}
$$

holds for some $C>0$, all $\xi=\left(\xi_{1}, \ldots, \xi_{n}\right)^{\top} \in \mathbb{R}^{n}$ and $x \in \bar{\Omega}$. We note that the assumptions on the domain $\Omega$ and the coefficients of $\mathcal{L}$ can be relaxed but it is not our aim to treat the most general setting here. We refer the reader to, e.g. $[28,38,42,50,55,65]$ for possible generalizations and to $[1,7,33,34,35]$ for recent work on non-smooth domains. On the other hand, we do not impose any conditions on the growth of derivatives of $a_{j k}$ at infinity; cf. the stronger assumptions in [52, Condition 3.1].

In the following we denote by $H^{s}(\Omega)$ and $H^{s}(\partial \Omega), s \geq 0$, the usual Sobolev spaces of order $s$ of $L^{2}$-functions on $\Omega$ and $\partial \Omega$, respectively. The Sobolev space $H^{-s}(\partial \Omega), s>0$, of negative order is defined as the dual space of $H^{s}(\partial \Omega)$; see, e.g. [50, Section 7.3] and [2]. The closure of $C_{0}^{\infty}(\Omega)$ in $H^{s}(\Omega)$ is denoted by $H_{0}^{s}(\Omega)$. For a function $f \in C^{\infty}(\bar{\Omega})$ we denote the trace by $\left.f\right|_{\partial \Omega}$ and we set

$$
\left.\frac{\partial f}{\partial \nu_{\mathcal{L}}}\right|_{\partial \Omega}:=\left.\sum_{j, k=1}^{n} a_{j k} \nu_{j} \frac{\partial f}{\partial x_{k}}\right|_{\partial \Omega}
$$

where $\nu(x)=\left(\nu_{1}(x), \ldots, \nu_{n}(x)\right)^{\top}$ is the unit vector at the point $x \in \partial \Omega$ pointing out of $\Omega$. Recall that, for all $s>\frac{3}{2}$, the mapping $C^{\infty}(\bar{\Omega}) \ni f \mapsto$ $\left\{\left.f\right|_{\partial \Omega},\left.\frac{\partial f}{\partial \nu_{\mathcal{L}}}\right|_{\partial \Omega}\right\}$ extends by continuity to a continuous surjective mapping

$$
H^{s}(\Omega) \ni f \mapsto\left\{\left.f\right|_{\partial \Omega},\left.\frac{\partial f}{\partial \nu_{\mathcal{L}}}\right|_{\partial \Omega}\right\} \in H^{s-1 / 2}(\partial \Omega) \times H^{s-3 / 2}(\partial \Omega),
$$

which admits a bounded right inverse. For $s=2$ the kernel of the mapping in $(4.2)$ is equal to $H_{0}^{2}(\Omega)$. 
In order to construct a quasi boundary triple for the maximal operator associated with $\mathcal{L}$ in $L^{2}(\Omega)$ in an " $H^{2}$ setting", we fix a suitable operator $T$ as the domain of the boundary mappings.

Definition 4.1. The differential operator $T f=\mathcal{L} f$ (understood in the distributional sense) is defined on the domain

$$
\operatorname{dom} T= \begin{cases}H^{2}(\Omega) & \text { if } \Omega \text { is bounded, } \\ \left\{f \in H^{1}(\Omega): \mathcal{L} f \in L^{2}(\Omega),\left.f\right|_{\Omega^{\prime}} \in H^{2}\left(\Omega^{\prime}\right)\right\} & \text { if } \Omega \text { is unbounded, }\end{cases}
$$

where in the unbounded case $\Omega^{\prime} \subset \Omega$ is a bounded subdomain of $\Omega$ with smooth boundary such that $\partial \Omega \subset \partial \Omega^{\prime}$.

In the unbounded case in Definition 4.1 we can choose, for instance, $\Omega^{\prime}=\Omega \cap B_{R}(0)$, where $B_{R}(0)=\left\{x \in \mathbb{R}^{n}:\|x\|<R\right\}$ and $R$ is big enough so that $\mathbb{R}^{n} \backslash \Omega \subset B_{R}(0)$. Since the condition $\mathcal{L} f \in L^{2}(\Omega)$ implies that $f \in$ $H_{\text {loc }}^{2}(\Omega)$ (see, e.g. [50, Theorem 2.3.2]), it is clear that the set on the righthand side of $\operatorname{dom} T$ in the case of unbounded $\Omega$ is independent of $\Omega^{\prime}$. We also note that, under the additional assumption that the coefficients $a_{j k}$ have bounded uniformly continuous derivatives, the domain of $T$ is globally $H^{2}$, cf. [8, Theorem 7.1 (i)].

In both cases ( $\Omega$ bounded or unbounded), functions $f$ in $\operatorname{dom} T$ are in $H^{2}$ in a neighbourhood of $\partial \Omega$, and hence $\left.f\right|_{\partial \Omega}$ and $\left.\frac{\partial f}{\partial \nu_{\mathcal{L}}}\right|_{\partial \Omega}$ are well defined and have values in $H^{3 / 2}(\partial \Omega)$ and $H^{1 / 2}(\partial \Omega)$, respectively. Define the Dirichlet, Neumann and minimal operator associated with $\mathcal{L}$ by

$$
\begin{aligned}
A_{\mathrm{D}} f=\mathcal{L} f, & \operatorname{dom} A_{\mathrm{D}}=\left\{f \in \operatorname{dom} T:\left.f\right|_{\partial \Omega}=0\right\}, \\
A_{\mathrm{N}} f=\mathcal{L} f, \quad \operatorname{dom} A_{\mathrm{N}} & =\left\{f \in \operatorname{dom} T:\left.\frac{\partial f}{\partial \nu_{\mathcal{L}}}\right|_{\partial \Omega}=0\right\}, \\
A f=\mathcal{L} f, & \operatorname{dom} A=\left\{f \in \operatorname{dom} T:\left.f\right|_{\partial \Omega}=0,\left.\frac{\partial f}{\partial \nu_{\mathcal{L}}}\right|_{\partial \Omega}=0\right\} .
\end{aligned}
$$

In the following theorem it is shown how a quasi boundary triple can be defined in the present situation. The self-adjointness of $A_{\mathrm{N}}$ in the case of an unbounded domain is shown in full detail, the remaining assertions are essentially a consequence of [10, Theorem 2.3].

Theorem 4.2. Let $\mathcal{L}$ be the uniformly elliptic differential expression from (4.1), let $T, A_{\mathrm{D}}, A_{\mathrm{N}}, A$ be the differential operators from above and define the boundary mappings

$$
\Gamma_{0} \hat{f}:=\left.\frac{\partial f}{\partial \nu_{\mathcal{L}}}\right|_{\partial \Omega} \quad \text { and } \quad \Gamma_{1} \hat{f}:=\left.f\right|_{\partial \Omega}, \quad \hat{f}=\left(\begin{array}{c}
f \\
T f
\end{array}\right), \quad f \in \operatorname{dom} T .
$$

Then $A$ is a densely defined closed symmetric operator in $L^{2}(\Omega)$, the operators $A_{\mathrm{N}}=\operatorname{ker} \Gamma_{0}$ and $A_{\mathrm{D}}=\operatorname{ker} \Gamma_{1}$ are self-adjoint extensions of $A$, and $\left\{L^{2}(\partial \Omega), \Gamma_{0}, \Gamma_{1}\right\}$ is a quasi boundary triple for $A^{*}$. Moreover,

$$
(T f, g)=\mathfrak{a}[f, g]-\left(\Gamma_{0} f, \Gamma_{1} g\right)
$$


holds for all $f, g \in \operatorname{dom} T$, where

$$
\mathfrak{a}[f, g]:=\int_{\Omega}\left(\sum_{j, k=1}^{n} a_{j k} \frac{\partial f}{\partial x_{k}} \frac{\partial \bar{g}}{\partial x_{j}}+a f \bar{g}\right), \quad f, g \in H^{1}(\Omega) .
$$

Proof. If $\Omega$ is bounded, the assertions in the theorem apart from (4.3) were proved in [10, Proposition 4.1]. The proof of (4.3) follows easily from known results; see also the proof below for the case where $\Omega$ is unbounded.

Now let $\Omega$ be unbounded. First we show that $A_{\mathrm{N}}$ as defined above is self-adjoint. Let the symmetric quadratic form $\mathfrak{a}[f, g]$ be as in the theorem. Because of the boundedness of the coefficients and the uniform ellipticity, this quadratic form can be compared with the form

$$
\mathfrak{a}_{0}[f, g]=\int_{\Omega} \operatorname{grad} f \cdot \overline{\operatorname{grad} g}
$$

which corresponds to the Laplace operator, namely, there exist constants $c_{1}, c_{2}>0$ and $d_{1}, d_{2} \in \mathbb{R}$ such that

$$
c_{1} \mathfrak{a}_{0}[f, f]+d_{1}\|f\|^{2} \leq \mathfrak{a}[f, f] \leq c_{2} \mathfrak{a}_{0}[f, f]+d_{2}\|f\|^{2} .
$$

Since $\|f\|^{2}+\mathfrak{a}_{0}[f, f]=\|f\|_{H^{1}(\Omega)}^{2}$, this implies that the form $\mathfrak{a}$ is closed and bounded from below. Hence, by [45, Theorem VI.2.1] there exists a selfadjoint operator $\widetilde{A}_{\mathrm{N}}$ in $L^{2}(\Omega)$ with $\operatorname{dom} \widetilde{A}_{\mathrm{N}} \subset \operatorname{dom} \mathfrak{a}=H^{1}(\Omega)$ which is bounded from below and represents the form $\mathfrak{a}$, i.e.

$$
\left(\widetilde{A}_{\mathrm{N}} f, g\right)=\mathfrak{a}[f, g]
$$

for all $f \in \operatorname{dom} \widetilde{A}_{\mathrm{N}}$ and $g \in H^{1}(\Omega)$.

We claim that the domain of $\widetilde{A}_{\mathrm{N}}$ is equal to

$$
\left\{f \in H^{1}(\Omega): \mathcal{L} f \in L^{2}(\Omega),\left.\frac{\partial f}{\partial \nu_{\mathcal{L}}}\right|_{\partial \Omega}=0\right\}
$$

and that $\widetilde{A}_{\mathrm{N}} f=\mathcal{L} f$ for $f \in \operatorname{dom} \widetilde{A}_{\mathrm{N}}$. In fact, let $f \in \operatorname{dom} \widetilde{A}_{\mathrm{N}}$. Then (4.5) is true in particular for $g \in C_{0}^{\infty}(\Omega)$, which implies that

$$
\left(\widetilde{A}_{\mathrm{N}} f, g\right)=\mathfrak{a}[f, g]=(f, \mathcal{L} g)=\langle\mathcal{L} f, g\rangle,
$$

where the last term is the application of the distribution $\mathcal{L} f$ to the test function $g$; the second equality follows from the definition of distributional derivatives. This implies that $\mathcal{L} f$ is a regular distribution and equals $\widetilde{A}_{\mathrm{N}} f \in$ $L^{2}(\Omega)$. The formula

$$
(\mathcal{L} u, v)=\mathfrak{a}[u, v]-\int_{\partial \Omega} \frac{\partial u}{\partial \nu_{\mathcal{L}}} \bar{v}
$$

is valid for all $u \in H^{1}(\Omega)$ such that $\mathcal{L} u \in L^{2}(\Omega)$ and all $v \in H^{1}(\Omega)$ such that one of the two functions has bounded support ${ }^{1}$. The derivative of $u$ in the

\footnotetext{
${ }^{1}$ Indeed, for $u, v \in H^{2}(\Omega)$ and bounded $\Omega$, formula (4.7) is well known. Since in this case $H^{2}(\Omega)$ is dense in $H_{\mathcal{L}}^{1}(\Omega):=\left\{w \in H^{1}(\Omega): \mathcal{L} w \in L^{2}(\Omega)\right\}$ equipped with the norm $\|w\|_{H^{1}}+$ $\|\mathcal{L} w\|_{L^{2}}$ and $\frac{\partial}{\partial \nu_{\mathcal{L}}}: H_{\mathcal{L}}^{1}(\Omega) \rightarrow H^{-1 / 2}(\partial \Omega)$ is continuous (see $[39,50]$ ), an approximation argument implies (4.7).
} 
integral is in $H^{-1 / 2}(\partial \Omega)$, the trace of $v$ is in $H^{1 / 2}(\partial \Omega)$; so the integral is understood as a dual pairing of $H^{-1 / 2}(\partial \Omega)$ and $H^{1 / 2}(\partial \Omega)$. Since boundary values of $H^{1}(\Omega)$-functions with bounded support exhaust the space $H^{1 / 2}(\partial \Omega)$, relations (4.5) and (4.7) with $u=f$ and $v=g$ yield that $\left.\frac{\partial f}{\partial \nu_{\mathcal{L}}}\right|_{\partial \Omega}=0$. Hence $f$ is in the set in (4.6). Conversely, let $f$ be in the set in (4.6). Then, by (4.7), we have $(\mathcal{L} f, g)=\mathfrak{a}[f, g]$ for all $g \in C^{\infty}(\Omega)$ with bounded support. This implies that $f \in \operatorname{dom} \widetilde{A}_{\mathrm{N}}$ and $\widetilde{A}_{\mathrm{N}} f=\mathcal{L} f$ by [45, Theorem VI.2.1 (iii)] since $\left\{g \in C^{\infty}(\Omega)\right.$ : supp $g$ bounded $\}$ is dense in $H^{1}(\Omega)$, which implies that it is a core of $\mathfrak{a}$.

We show that functions in $\operatorname{dom} \widetilde{A}_{\mathrm{N}}$ are in $H^{2}$ in a neighbourhood of $\partial \Omega$. Let $R>0$ be such that $\mathbb{R}^{n} \backslash \Omega \subset B_{R}(0)$ and set $\Omega^{\prime}:=\Omega \cap B_{R}(0)$. Moreover, choose a $C^{\infty}$-function $\varphi$ defined on $\Omega$ such that $\operatorname{supp} \varphi \subset \Omega^{\prime}$, that $\varphi(x)=1$ in a neighbourhood of $\partial \Omega$ and that $\varphi(x)=0$ in a neighbourhood of $S_{R}(0):=\left\{x \in \mathbb{R}^{n}:\|x\|=R\right\}$. Let $f$ be in dom $\widetilde{A}_{\mathrm{N}}$, i.e. in the set in (4.6). We want to show that $\varphi f \in \operatorname{dom} \widetilde{A}_{\mathrm{N}}$. Clearly, $\varphi f \in H^{1}(\Omega)$. Since

$$
\mathcal{L}(\varphi f)=\varphi(\mathcal{L} f)-\sum_{j, k=1}^{n}\left[2 a_{j k} \frac{\partial \varphi}{\partial x_{j}} \frac{\partial f}{\partial x_{k}}+f \frac{\partial a_{j k}}{\partial x_{j}} \frac{\partial \varphi}{\partial x_{k}}+a_{j k} f \frac{\partial^{2} \varphi}{\partial x_{j} \partial x_{k}}\right]
$$

$f \in H^{1}(\Omega)$ and the derivatives of $a_{j k}$ and $\varphi$ are uniformly bounded on the bounded set $\operatorname{supp} \varphi$, we can deduce that $\mathcal{L}(\varphi f) \in L^{2}(\Omega)$. The validity of the boundary condition $\left.\frac{\partial(\varphi f)}{\partial \nu \mathcal{L}}\right|_{\partial \Omega}=0$ is clear from the fact that $\varphi(x)=1$ in a neighbourhood of $\partial \Omega$. It follows that $\varphi f$ is in the set in (4.6) and hence in $\operatorname{dom} \widetilde{A}_{\mathrm{N}}$. Now define a quadratic form $\mathfrak{a}_{\Omega^{\prime}, \mathrm{N}, \mathrm{D}}$ in $L^{2}\left(\Omega^{\prime}\right)$ by the formula in (4.4) with domain

$$
\operatorname{dom} \mathfrak{a}_{\Omega^{\prime}, \mathrm{N}, \mathrm{D}}=\left\{h \in H^{1}\left(\Omega^{\prime}\right):\left.f\right|_{S_{R}(0)}=0\right\} .
$$

This form defines a self-adjoint operator $A_{\Omega^{\prime}, \mathrm{N}, \mathrm{D}}$ :

$$
A_{\Omega^{\prime}, \mathrm{N}, \mathrm{D}} h=\mathcal{L} h, \quad \operatorname{dom} A_{\Omega^{\prime}, \mathrm{N}, \mathrm{D}}=\left\{h \in H^{2}\left(\Omega^{\prime}\right):\left.h\right|_{S_{R}(0)}=0,\left.\frac{\partial h}{\partial \nu_{\mathcal{L}}}\right|_{\partial \Omega}=0\right\} .
$$

Since $f \in \operatorname{dom} \widetilde{A}_{\mathrm{N}}$ and any function $g$ in dom $\mathfrak{a}_{\Omega^{\prime}, \mathrm{N}, \mathrm{D}}$ can be extended by 0 to a function $\widetilde{g}$ in $H^{1}(\Omega)$, we have

$$
\left(\left.\left(\widetilde{A}_{\mathrm{N}} f\right)\right|_{\Omega^{\prime}}, g\right)_{L^{2}\left(\Omega^{\prime}\right)}=\left(\widetilde{A}_{\mathrm{N}} f, \widetilde{g}\right)_{L^{2}(\Omega)}=\mathfrak{a}[f, \widetilde{g}]=\mathfrak{a}_{\Omega^{\prime}, \mathrm{N}, \mathrm{D}}\left[\left.f\right|_{\Omega^{\prime}}, g\right]
$$

for all $g \in \operatorname{dom} \mathfrak{a}_{\Omega^{\prime}, \mathrm{N}, \mathrm{D}}$. By [45, Theorem VI.2.1 (iii)] this implies that $\left.f\right|_{\Omega^{\prime}} \in$ $\operatorname{dom} A_{\Omega^{\prime}, \mathrm{N}, \mathrm{D}}$ and hence $\left.f\right|_{\Omega^{\prime}} \in H^{2}\left(\Omega^{\prime}\right)$.

It follows that

$$
\begin{aligned}
\operatorname{dom} \widetilde{A}_{\mathrm{N}} & =\left\{f \in H^{1}(\Omega): \mathcal{L} f \in L^{2}(\Omega),\left.\frac{\partial f}{\partial \nu_{\mathcal{L}}}\right|_{\partial \Omega}=0\right\} \\
& =\left\{f \in H^{1}(\Omega): \mathcal{L} f \in L^{2}(\Omega),\left.\frac{\partial f}{\partial \nu_{\mathcal{L}}}\right|_{\partial \Omega}=0,\left.f\right|_{\Omega^{\prime}} \in H^{2}\left(\Omega^{\prime}\right)\right\} \\
& =\left\{f \in \operatorname{dom} T:\left.\frac{\partial f}{\partial \nu_{\mathcal{L}}}\right|_{\partial \Omega}=0\right\}=\operatorname{dom} A_{\mathrm{N}}
\end{aligned}
$$


and that $A_{\mathrm{N}}=\widetilde{A}_{\mathrm{N}}$ is a self-adjoint operator in $L^{2}(\Omega)$. With a similar reasoning and using the quadratic form a restricted to $H_{0}^{1}(\Omega)$ one can show that $A_{\mathrm{D}}$ is a self-adjoint operator in $L^{2}(\Omega)$.

Next we show, with the help of $\left[10\right.$, Theorem 2.3], that $\left\{L^{2}(\partial \Omega), \Gamma_{0}, \Gamma_{1}\right\}$ is a quasi boundary triple. It follows from the considerations before the statement of the current theorem that $\Gamma_{0}$ and $\Gamma_{1}$ are well defined. Moreover,

$$
\operatorname{ran} \Gamma=\operatorname{ran}\left(\begin{array}{l}
\Gamma_{0} \\
\Gamma_{1}
\end{array}\right)=H^{1 / 2}(\partial \Omega) \times H^{3 / 2}(\partial \Omega)
$$

(see, e.g. [50, Theorem 1.8.3]), which is dense in $L^{2}(\partial \Omega) \times L^{2}(\partial \Omega)$. In order to show Green's identity, we first show the identity (4.3). Let $\Omega^{\prime}$ and $\varphi$ be as above and set $\psi:=1-\varphi$. If $f, g \in \operatorname{dom} T$, then $\left.(\varphi f)\right|_{\Omega^{\prime}},\left.(\varphi g)\right|_{\Omega^{\prime}} \in H^{2}\left(\Omega^{\prime}\right)$ and $\psi f, \psi g \in \operatorname{dom} A_{\mathrm{N}}$. Using (4.7) and (4.5) we obtain

$$
\begin{aligned}
& (T f, g)_{L^{2}(\Omega)}=(T f, \varphi g)_{L^{2}\left(\Omega^{\prime}\right)}+(T(\varphi f), \psi g)_{L^{2}\left(\Omega^{\prime}\right)}+(T(\psi f), \psi g)_{L^{2}(\Omega)} \\
& =\mathfrak{a}[f, \varphi g]-\int_{\partial \Omega} \frac{\partial f}{\partial \nu_{\mathcal{L}}} \overline{\varphi g}+\mathfrak{a}[\varphi f, \psi g]-\int_{\partial \Omega} \frac{\partial(\varphi f)}{\partial \nu_{\mathcal{L}}} \overline{\psi g}+\mathfrak{a}[\psi f, \psi g] \\
& =\mathfrak{a}[f, g]-\left(\Gamma_{0} f, \Gamma_{1} g\right)_{L^{2}(\partial \Omega)}
\end{aligned}
$$

since $\varphi(x)=1$ and $\psi(x)=0$ in a neighbourhood of $\partial \Omega$, which proves (4.3). The abstract Green identity (3.1) follows immediately from this and the symmetry of $\mathfrak{a}$. Now we can apply [10, Theorem 2.3] to obtain that $A$ is a closed, symmetric operator and that $\left\{L^{2}(\partial \Omega), \Gamma_{0}, \Gamma_{1}\right\}$ is a quasi boundary triple. Moreover, since $T$ is an operator, we conclude that $T^{*}=A$ is densely defined.

Observe that, for the quasi boundary triple in Theorem 4.2, we have

$$
\mathcal{G}_{0}=\operatorname{ran} \Gamma_{0}=H^{1 / 2}(\partial \Omega) \quad \text { and } \quad \mathcal{G}_{1}=\operatorname{ran} \Gamma_{1}=H^{3 / 2}(\partial \Omega) .
$$

We also note that the triple $\left\{L^{2}(\partial \Omega), \Gamma_{0}, \Gamma_{1}\right\}$ is not a generalized boundary triple or a boundary relation in the sense of $[24,27]$ and we refer to $[20$, 39] for a modified approach that leads to an ordinary boundary triple for $A^{*}$. One of the advantages of the quasi boundary triple in Theorem 4.2 is that the corresponding Weyl function is the inverse of the usual Dirichletto-Neumann map, whereas the Weyl function corresponding to the ordinary boundary triple from [20,39] (which differs by an unbounded constant from the Dirichlet-to-Neumann map) is more difficult to interpret; see also [9, Proposition 4.1]. The $\gamma$-field corresponding to the quasi boundary triple from Theorem 4.2 is the Poisson operator for the Neumann problem associated with $\mathcal{L}$. This is summarized in the following proposition, whose proof is clear from the definitions of $\gamma(\lambda)$ and $M(\lambda)$.

Proposition 4.3. Let $\operatorname{dom} T$ be as in Definition 4.1. For $\varphi \in H^{1 / 2}(\partial \Omega)$ and $\lambda \in \rho\left(A_{\mathrm{N}}\right)$ denote the unique solution of

$$
\mathcal{L} h=\lambda h,\left.\quad \frac{\partial h}{\partial \nu_{\mathcal{L}}}\right|_{\partial \Omega}=\varphi
$$


in $\operatorname{dom} T$ by $f_{\lambda}(\varphi)$. Then the $\gamma$-field $\gamma$ and Weyl function $M$ associated with the quasi boundary triple $\left\{L^{2}(\partial \Omega), \Gamma_{0}, \Gamma_{1}\right\}$ in Theorem 4.2 are given by

$$
\begin{array}{rlrl}
\gamma(\lambda): H^{1 / 2}(\partial \Omega) & \rightarrow L^{2}(\Omega), & \varphi & \mapsto f_{\lambda}(\varphi) \in \operatorname{ker}(T-\lambda), \\
M(\lambda): H^{1 / 2}(\partial \Omega) & \rightarrow H^{3 / 2}(\partial \Omega),\left.\quad \varphi \mapsto f_{\lambda}(\varphi)\right|_{\partial \Omega} .
\end{array}
$$

It is known from [50,63] that $M(\lambda)$ can be extended to a bounded operator acting between various Sobolev spaces. For the convenience of the reader we give a short proof based on a duality and interpolation argument.

Lemma 4.4. Let $s \in\left[-\frac{3}{2}, \frac{1}{2}\right]$ and $\lambda \in \rho\left(A_{\mathrm{N}}\right)$. Then $M(\lambda)$ can be extended to a bounded operator from $H^{s}(\partial \Omega)$ to $H^{s+1}(\partial \Omega)$. Moreover, the closure $\overline{M(\lambda)}$ in $L^{2}(\partial \Omega)$ is a compact operator in $L^{2}(\partial \Omega)$ with $\operatorname{ran}(\overline{M(\lambda)}) \subset H^{1}(\partial \Omega)$.

Proof. Denote by $\langle\cdot, \cdot\rangle_{t}$ the dual pairing of $H^{t}(\partial \Omega)$ and $H^{-t}(\partial \Omega)$ for $t \geq 0$, i.e. $\langle x, y\rangle_{t}$ is defined for $x \in H^{t}(\partial \Omega)$ and $y \in H^{-t}(\partial \Omega),\langle\cdot, \cdot\rangle_{t}$ is linear in the first and semi-linear in the second component and satisfies

$$
\langle x, y\rangle_{t}=(x, y) \quad \text { for } x \in H^{t}(\partial \Omega), y \in L^{2}(\partial \Omega),
$$

where $(\cdot, \cdot)$ denotes the inner product in $L^{2}(\partial \Omega)$.

In the following, let $\lambda \in \rho\left(A_{\mathrm{N}}\right)$. Proposition $3.3(\mathrm{v})$ implies that $M(\lambda)$ is closable in $L^{2}(\partial \Omega)$ and Proposition 3.3 (iii) shows that it maps $H^{1 / 2}(\partial \Omega)$ into $H^{3 / 2}(\partial \Omega)$. Therefore $M(\lambda)$ is closed and hence bounded from $H^{1 / 2}(\partial \Omega)$ to $H^{3 / 2}(\partial \Omega)$.

The Banach space adjoint $(M(\bar{\lambda}))^{\prime}$ of $M(\bar{\lambda})$ is a bounded operator from $H^{-3 / 2}(\partial \Omega)$ to $H^{-1 / 2}(\partial \Omega)$, where $(M(\bar{\lambda}))^{\prime}$ is defined by

$$
\left\langle x,(M(\bar{\lambda}))^{\prime} y\right\rangle_{1 / 2}=\langle M(\bar{\lambda}) x, y\rangle_{3 / 2}, \quad x \in H^{1 / 2}(\partial \Omega), y \in H^{-3 / 2}(\partial \Omega) .
$$

Proposition $3.3(\mathrm{v})$ yields that $(M(\bar{\lambda}) x, y)=(x, M(\lambda) y)$ for $x, y \in H^{1 / 2}(\partial \Omega)$. Combining this relation with (4.8) and (4.9) we obtain, for $x, y \in H^{1 / 2}(\partial \Omega)$, that

$$
\begin{aligned}
\langle x, M(\lambda) y\rangle_{1 / 2} & =(x, M(\lambda) y)=(M(\bar{\lambda}) x, y) \\
& =\langle M(\bar{\lambda}) x, y\rangle_{3 / 2}=\left\langle x,(M(\bar{\lambda}))^{\prime} y\right\rangle_{1 / 2} .
\end{aligned}
$$

This implies that $M(\lambda) y=(M(\bar{\lambda}))^{\prime} y$ for $y \in H^{1 / 2}(\partial \Omega)$. Hence the bounded operator $(M(\bar{\lambda}))^{\prime}: H^{-3 / 2}(\partial \Omega) \rightarrow H^{-1 / 2}(\partial \Omega)$ is an extension of the mapping $M(\lambda): H^{1 / 2}(\partial \Omega) \rightarrow H^{3 / 2}(\partial \Omega)$. Now interpolation (see, e.g. [50, Theorems 5.1 and 7.7]) implies that

$$
\left.(M(\bar{\lambda}))^{\prime}\right|_{H^{s}(\partial \Omega)}: H^{s}(\partial \Omega) \rightarrow H^{s+1}(\partial \Omega)
$$

is bounded for $s \in\left[-\frac{3}{2}, \frac{1}{2}\right]$.

Since $\overline{M(\lambda)}=\left.(M(\bar{\lambda}))^{\prime}\right|_{L^{2}(\partial \Omega)}$, we can deduce from (4.10) that $\overline{M(\lambda)}$ is bounded from $L^{2}(\partial \Omega)$ to $H^{1}(\partial \Omega)$. Together with the compactness of the embedding of $H^{1}(\partial \Omega)$ into $L^{2}(\partial \Omega)$ (see, e.g. [65, Theorem 7.10]) this shows that $\overline{M(\lambda)}$ is a compact operator in $L^{2}(\partial \Omega)$. 
In [10] and [12] quasi boundary triples for elliptic operators were also studied in the framework of the Beals space $\mathcal{D}_{1}(\Omega)$ when $\Omega$ is bounded with a smooth boundary. In this setting sufficient conditions on the parameter $\Theta$ in $L^{2}(\partial \Omega)$ that ensure self-adjointness of the corresponding elliptic operator

$$
A_{\Theta}=\mathcal{L} \uparrow\left\{f \in \mathcal{D}_{1}(\Omega): \Gamma \hat{f} \in \Theta\right\}
$$

were obtained in [10, Theorem 4.8]. The next result gives a sufficient condition on $\Theta$ in the $H^{2}$-framework, which also covers a large class of Robin type boundary conditions; cf. Corollary 4.6 below. We note that $\Omega$ is allowed to be unbounded but $\partial \Omega$ is assumed to be compact and smooth.

Theorem 4.5. Let $\left\{L^{2}(\partial \Omega), \Gamma_{0}, \Gamma_{1}\right\}$ be the quasi boundary triple from Theorem 4.2 and $\Gamma=\left(\Gamma_{0}, \Gamma_{1}\right)^{\top}$. Let $\Theta$ be a self-adjoint relation in $L^{2}(\partial \Omega)$ such that $0 \notin \sigma_{\text {ess }}(\Theta)$ and

$$
\Theta^{-1}\left(H^{1}(\partial \Omega)\right) \subset H^{1 / 2}(\partial \Omega) .
$$

Then the realization $A_{\Theta}=\mathcal{L} \uparrow\{f \in \operatorname{dom} T: \Gamma \hat{f} \in \Theta\}$ is self-adjoint in $L^{2}(\Omega)$. In particular, if $B$ is a bounded operator in $L^{2}(\partial \Omega)$ with $B\left(H^{1}(\partial \Omega)\right) \subset$ $H^{1 / 2}(\partial \Omega)$, then the realization

$$
A_{B^{-1}} f=\mathcal{L} f, \quad \operatorname{dom} A_{B^{-1}}=\left\{f \in \operatorname{dom} T: B\left(\left.f\right|_{\partial \Omega}\right)=\left.\frac{\partial f}{\partial \nu_{\mathcal{L}}}\right|_{\partial \Omega}\right\},
$$

is a self-adjoint operator in $L^{2}(\Omega)$.

Proof. We can apply Theorem 3.11 and Remark 3.12 since $\operatorname{ran} \overline{M(\lambda)} \subset$ $H^{1}(\partial \Omega)$ for all $\lambda \in \rho\left(A_{\mathrm{N}}\right)$ by Lemma 4.4 .

The next corollary is an immediate consequence of Theorem 4.5. In includes, in particular, classical Robin boundary conditions.

Corollary 4.6. Let $\beta \in C^{1}(\partial \Omega)$ be a real-valued function on $\partial \Omega$ and $k \in$ $C^{1}(\partial \Omega \times \partial \Omega)$ a symmetric kernel on $\partial \Omega$, i.e. $k(x, y)=\overline{k(y, x)}$ for $x, y \in \partial \Omega$. Then the realization

$$
A_{B^{-1}} f=\mathcal{L} f, \quad \operatorname{dom} A_{B^{-1}}=\left\{f \in \operatorname{dom} T: B\left(\left.f\right|_{\partial \Omega}\right)=\left.\frac{\partial f}{\partial \nu_{\mathcal{L}}}\right|_{\partial \Omega}\right\}
$$

where

$$
(B \varphi)(x)=\beta(x) \varphi(x)+\int_{\partial \Omega} k(x, y) \varphi(y) d y, \quad \varphi \in L^{2}(\partial \Omega),
$$

is a self-adjoint operator in $L^{2}(\Omega)$.

Before we continue to investigate resolvent differences of self-adjoint realizations of $\mathcal{L}$, we need the following general lemma on the singular values of operators mapping into Sobolev spaces; see also [12] for a special case. The proof is essentially an application of results on the asymptotic behaviour of eigenvalues of the Laplace-Beltrami operator on compact manifolds; for similar ideas see the proof of [3, Proposition 5.4.1]. 
Lemma 4.7. Let $\Sigma$ be an $(n-1)$-dimensional compact manifold without boundary, let $\mathcal{K}$ be a Hilbert space and $B \in \mathcal{B}\left(\mathcal{K}, H^{r_{1}}(\Sigma)\right)$ with $\operatorname{ran} B \subset H^{r_{2}}(\Sigma)$ where $r_{2}>r_{1} \geq 0$. Then $B$ is compact and its singular values $s_{k}$ satisfy

$$
s_{k}(B)=\mathrm{O}\left(k^{-\frac{r_{2}-r_{1}}{n-1}}\right), \quad k \rightarrow \infty,
$$

i.e. $B \in \mathfrak{S}_{\frac{n-1}{r_{2}-r_{1}}, \infty}\left(\mathcal{K}, H^{r_{1}}(\Sigma)\right)$ and, in particular, $B \in \mathfrak{S}_{p}\left(\mathcal{K}, H^{r_{1}}(\Sigma)\right)$ for $p>\frac{n-1}{r_{2}-r_{1}}$.

Proof. Let $\Lambda_{r_{1}, r_{2}}:=\left(I-\Delta_{\mathrm{LB}}^{\Sigma}\right)^{\frac{r_{2}-r_{1}}{2}}$, where $\Delta_{\mathrm{LB}}^{\Sigma}$ is the Laplace-Beltrami operator on $\Sigma$. The operator $\Lambda_{r_{1}, r_{2}}$ is an isometric isomorphism from $H^{r_{2}}(\Sigma)$ onto $H^{r_{1}}(\Sigma)$. From [3, (5.39) and the text below] we obtain, for the asymptotics of the eigenvalues, $\lambda_{k}\left(I-\Delta_{\mathrm{LB}}^{\Sigma}\right) \sim C k^{\frac{2}{n-1}}$ with some constant $C$. This implies that

$$
s_{k}\left(\Lambda_{r_{1}, r_{2}}^{-1}\right)=\mathrm{O}\left(k^{-\frac{r_{2}-r_{1}}{n-1}}\right), \quad k \rightarrow \infty .
$$

We can write $B$ in the form

$$
B=\Lambda_{r_{1}, r_{2}}^{-1}\left(\Lambda_{r_{1}, r_{2}} B\right) .
$$

The operator $B$ is closed as an operator from $\mathcal{K}$ into $H^{r_{1}}(\Sigma)$, hence also closed as an operator from $\mathcal{K}$ into $H^{r_{2}}(\Sigma)$, which implies that it is bounded from $\mathcal{K}$ into $H^{r_{2}}(\Sigma)$. Therefore the operator $\Lambda_{r_{1}, r_{2}} B$ is bounded from $\mathcal{K}$ into $H^{r_{1}}(\Sigma)$, and hence the assertions follow from (4.11).

The next result is essentially a consequence of the previous lemma, Lemma 4.4 and general properties of the $\gamma$-field and the Weyl function established in Section 3.1. In a slightly different setup items (i) and (iv) appeared in [14, Proposition 3.3]; however, for the convenience of the reader, we give the short proofs of all items.

Proposition 4.8. Let $\left\{L^{2}(\partial \Omega), \Gamma_{0}, \Gamma_{1}\right\}$ be the quasi boundary triple from Theorem 4.2. Then, for $\lambda \in \rho\left(A_{\mathrm{N}}\right)$, the associated $\gamma$-field $\gamma$, the Weyl function $M$ and the closures $\overline{M(\lambda)}, \overline{\operatorname{Im} M(\lambda)}$ satisfy

(i) $\gamma(\lambda)^{*} \in \mathfrak{S}_{\frac{2(n-1)}{3}, \infty}\left(L^{2}(\Omega), L^{2}(\partial \Omega)\right)$;

(ii) $M(\lambda) \in \mathfrak{S}_{n-1, \infty}\left(H^{1 / 2}(\partial \Omega)\right)$;

(iii) $\overline{\operatorname{Im} M(\lambda)} \in \mathfrak{S}_{\frac{n-1}{3}, \infty}\left(L^{2}(\partial \Omega)\right)$;

(iv) $\overline{M(\lambda)} \in \mathfrak{S}_{n-1, \infty}\left(L^{2}(\partial \Omega)\right)$.

Proof. Assertion (i) follows from Lemma 4.7 with $r_{1}=0$ and $r_{2}=\frac{3}{2}$ since $\gamma(\lambda)^{*}$ is a bounded operator from $\mathcal{K}=L^{2}(\Omega)$ to $L^{2}(\partial \Omega)$ with $\operatorname{ran} \Gamma_{1} \subset$ $H^{3 / 2}(\partial \Omega)$ by Proposition 3.3 (ii).

(ii) By Lemma 4.4, the operator $M(\lambda), \lambda \in \rho\left(A_{\mathrm{N}}\right)$, is bounded as an operator in $H^{1 / 2}(\partial \Omega)$. Hence Lemma 4.7 applied with $\mathcal{K}=H^{1 / 2}(\partial \Omega), r_{1}=\frac{1}{2}$ and $r_{2}=\frac{3}{2}$ yields the assertion.

(iii) From Proposition $3.3(\mathrm{v})$ we obtain the relation

$$
\overline{\operatorname{Im} M(\lambda)}=(\operatorname{Im} \lambda) \gamma(\lambda)^{*} \overline{\gamma(\lambda)} \text {. }
$$


It follows from (i) and Lemma 2.3 (iii) that the right-hand side is contained in $\mathfrak{S}_{\frac{2(n-1)}{3}, \infty} \cdot \mathfrak{S}_{\frac{2(n-1)}{3}, \infty}=\mathfrak{S}_{\frac{n-1}{3}, \infty}$.

(iv) The statement follows from Lemmas 4.4 and 4.7 with $\mathcal{K}=L^{2}(\partial \Omega)$, $r_{1}=0$ and $r_{2}=1$.

Remark 4.9. It is not difficult to check that $\left\{L^{2}(\partial \Omega), \Gamma_{1},-\Gamma_{0}\right\}$ is also a quasi boundary triple for the operator $A^{*}$. The corresponding Weyl function $\widehat{M}$ is - up to a minus sign — the Dirichlet-to-Neumann map from $H^{3 / 2}(\partial \Omega)$ to $H^{1 / 2}(\partial \Omega)$, i.e. for $\lambda \in \rho\left(A_{\mathrm{D}}\right)$ the operator $\widehat{M}(\lambda)$ maps the Dirichlet boundary value $\left.f_{\lambda}(\varphi)\right|_{\partial \Omega}$ of the solution $f_{\lambda}(\varphi) \in \operatorname{dom} T$ of $\mathcal{L} h=\lambda h,\left.h\right|_{\partial \Omega}=\varphi$, onto the (minus) Neumann boundary value $-\left.\frac{\partial f_{\lambda}(\varphi)}{\partial \nu_{\mathcal{L}}}\right|_{\partial \Omega}$. One of the main reasons that we do not use the quasi boundary triple $\left\{L^{2}(\partial \Omega), \Gamma_{1},-\Gamma_{0}\right\}$ here is that the values of the corresponding Weyl function $\widehat{M}$ are unbounded operators in $L^{2}(\partial \Omega)$.

\subsection{Spectral estimates for resolvent differences of self-adjoint elliptic oper- ators on bounded and exterior domains}

Throughout this section let $\left\{L^{2}(\partial \Omega), \Gamma_{0}, \Gamma_{1}\right\}$ be the quasi boundary triple from Theorem 4.2 with corresponding $\gamma$-field and Weyl function from Proposition 4.3. If $\Omega$ is unbounded, let $\Omega^{\prime}$ be as in Definition 4.1 ; if $\Omega$ is bounded, set $\Omega^{\prime}:=\Omega$. For a linear relation $\Theta$ in $L^{2}(\partial \Omega)$ the corresponding realization $A_{\Theta}$ of $\mathcal{L}$ is given by

$$
\begin{aligned}
A_{\Theta} f & =\mathcal{L} f \\
\operatorname{dom} A_{\Theta} & =\left\{f \in H^{1}(\Omega): \mathcal{L} f \in L^{2}(\Omega),\left.f\right|_{\Omega^{\prime}} \in H^{2}\left(\Omega^{\prime}\right),\left(\begin{array}{c}
\left.\frac{\partial f}{\partial \nu_{\mathcal{L}}}\right|_{\partial \Omega} \\
\left.f\right|_{\partial \Omega}
\end{array}\right) \in \Theta\right\} ;
\end{aligned}
$$

cf. (3.2), (3.3) and Theorem 4.2. A sufficient condition for the self-adjointness of $A_{\Theta}$ was given in Theorem 4.5. In the following, we apply the general results from Section 3.3 to resolvent differences of self-adjoint realizations of the elliptic differential expression $\mathcal{L}$ in $L^{2}(\Omega)$. The statements in the next three theorems are consequences of Proposition 4.8 and Theorems 3.13, 3.19 and 3.20 , respectively. Similar results were proved in [11, Theorem 6.27] and [14, Corollary 3.9 (i)]. In the case of classical Robin boundary conditions with a coefficient that satisfies certain smoothness assumptions the asymptotics of the singular values was determined in $[40,41,43]$.

Theorem 4.10. Let $A_{\mathrm{N}}$ be the Neumann operator associated with $\mathcal{L}$ and let $\Theta$ be a self-adjoint relation in $L^{2}(\partial \Omega)$ such that $0 \notin \sigma_{\mathrm{ess}}(\Theta)$ and $A_{\Theta}$ is a self-adjoint operator. Then, for all $\lambda \in \rho\left(A_{\Theta}\right) \cap \rho\left(A_{\mathrm{N}}\right)$, the singular values $s_{k}$ of the resolvent difference

$$
\left(A_{\Theta}-\lambda\right)^{-1}-\left(A_{\mathrm{N}}-\lambda\right)^{-1}
$$

satisfy $s_{k}=\mathrm{O}\left(k^{-\frac{3}{n-1}}\right), k \rightarrow \infty$, and the expression in $(4.12)$ is in $\mathfrak{S}_{p}\left(L^{2}(\Omega)\right)$ for all $p>\frac{n-1}{3}$. 
Proof. By Proposition $4.8(\mathrm{i})$ we have $\gamma(\lambda)^{*} \in \mathfrak{S}_{\frac{2(n-1)}{3}, \infty}\left(L^{2}(\Omega), L^{2}(\partial \Omega)\right)$. Hence we can apply Theorem 3.13, which yields that the resolvent difference in (4.12) belongs to

$$
\mathfrak{S}_{\frac{2(n-1)}{3}, \infty} \cdot \mathfrak{S}_{\frac{2(n-1)}{3}, \infty}=\mathfrak{S}_{\frac{n-1}{3}, \infty} \subset \mathfrak{S}_{p}, \quad p>\frac{n-1}{3},
$$

where we used Lemma 2.3 (iii) and (ii).

As an immediate consequence of Theorem 4.10 we obtain that the essential spectra of $A_{\Theta}$ and $A_{\mathrm{N}}$ coincide, $\sigma_{\text {ess }}\left(A_{\Theta}\right)=\sigma_{\text {ess }}\left(A_{\mathrm{N}}\right)$. In the case of a bounded domain these sets are empty, in the unbounded case the following proposition shows how close eigenvalues of $A_{\Theta}$ have to be to eigenvalues of $A_{\mathrm{N}}$.

Proposition 4.11. Let $\Omega$ be unbounded, let $A_{\mathrm{N}}$ be the Neumann operator associated with $\mathcal{L}$ and let $\Theta$ be a self-adjoint relation in $L^{2}(\partial \Omega)$ such that $0 \notin \sigma_{\mathrm{ess}}(\Theta)$ and $A_{\Theta}$ is a self-adjoint operator. If $\lambda_{k}, k=1,2, \ldots$, are isolated eigenvalues of $A_{\Theta}$ converging to some $\gamma \in \mathbb{R}$, then there exist numbers $\mu_{k}, k=1,2, \ldots$, which are isolated eigenvalues of $A_{\mathrm{N}}$ or equal to $\gamma$ (where the number $\gamma$ may appear arbitrarily many times but an eigenvalue only up to its multiplicity) such that

$$
\sum_{k=1}^{\infty}\left|\lambda_{k}-\mu_{k}\right|^{p}<\infty \quad \text { for all } p>\frac{n-1}{3}, p \geq 1 .
$$

Proof. The spectrum of $A_{\mathrm{N}}$ is bounded from below, which follows from (4.3) and the ellipticity of $\mathcal{L}$. Hence also the essential spectrum of $A_{\Theta}$ is bounded from below, and we can choose a number $\lambda \in \mathbb{R} \cap \rho\left(A_{\mathrm{N}}\right) \cap \rho\left(A_{\Theta}\right)$. Because of Theorem 4.10 we can apply $\left[44\right.$, Theorem II] to the operators $\left(A_{\mathrm{N}}-\lambda\right)^{-1}$ and $\left(A_{\Theta}-\lambda\right)^{-1}$, which yields that there exist extended enumerations $\left(\alpha_{k}\right)$ and $\left(\beta_{k}\right)$ of the isolated eigenvalues of $\left(A_{\mathrm{N}}-\lambda\right)^{-1}$ and $\left(A_{\Theta}-\lambda\right)^{-1}$, respectively, such that

$$
\sum_{k=1}^{\infty}\left|\beta_{k}-\alpha_{k}\right|^{p} \leq\left\|\left(A_{\Theta}-\lambda\right)^{-1}-\left(A_{\mathrm{N}}-\lambda\right)^{-1}\right\|_{\mathfrak{S}_{p}\left(L^{2}(\Omega)\right)}^{p}
$$

for $p>(n-1) / 3, p \geq 1$; by "extended enumeration" a sequence is meant that contains all isolated eigenvalues of an operator according to their multiplicities plus endpoints of the essential spectrum taken arbitrarily many times. There exist indices $j_{k}$ such that $\frac{1}{\lambda_{k}-\lambda}=\beta_{j_{k}}$. The corresponding values $\alpha_{j_{k}}$ can be written as $\alpha_{j_{k}}=\frac{1}{\mu_{k}-\lambda}$, where the $\mu_{k}$ are either isolated eigenvalues of $A_{\mathrm{N}}$ or endpoints of the essential spectrum. Now the estimate (4.14) implies that

$$
\sum_{k=1}^{\infty}\left|\frac{1}{\lambda_{k}-\lambda}-\frac{1}{\mu_{k}-\lambda}\right|^{p}<\infty
$$

Since $\lambda_{k} \rightarrow \gamma$, we must have $\mu_{k} \rightarrow \gamma$. Writing the difference of fractions as a single fraction and observing that the denominators converge to $\gamma-\lambda \neq 0$, we can deduce the validity of (4.13). 
If $n=2$ or $n=3$, then a trace formula is valid, which is stated in the next corollary and follows directly from Corollary 3.16. Under different assumptions trace formulae for resolvent power differences were shown in [14, Corollary 3.9].

Corollary 4.12. Let the assumptions be as in Theorem 4.10 and assume, in addition, that $n=2$ or $n=3$. Then the resolvent difference in (4.12) is a trace class operator and

$$
\operatorname{tr}\left(\left(A_{\Theta}-\lambda\right)^{-1}-\left(A_{\mathrm{N}}-\lambda\right)^{-1}\right)=\operatorname{tr}\left(\overline{M^{\prime}(\lambda)}(\Theta-\overline{M(\lambda)})^{-1}\right)
$$

holds for all $\lambda \in \rho\left(A_{\Theta}\right) \cap \rho\left(A_{\mathrm{N}}\right)$.

In the case $n=2$ or $n=3$ in the above corollary the wave operators of the pair $\left\{A_{\mathrm{N}}, A_{\Theta}\right\}$ exist and are complete (see, e.g. [45, Theorem X.4.12]) and hence the absolutely continuous parts of $A_{\mathrm{N}}$ and $A_{\Theta}$ are unitarily equivalent and the absolutely continuous spectra of $A_{\mathrm{N}}$ and $A_{\Theta}$ coincide.

The statement in the next theorem is a well known result from [16], but follows also easily from Theorem 3.19.

Theorem 4.13. Let $A_{\mathrm{N}}$ and $A_{\mathrm{D}}$ be the Neumann and Dirichlet operator associated with $\mathcal{L}$. Then, for all $\lambda \in \rho\left(A_{\mathrm{D}}\right) \cap \rho\left(A_{\mathrm{N}}\right)$, the singular values $s_{k}$ of the resolvent difference

$$
\left(A_{\mathrm{D}}-\lambda\right)^{-1}-\left(A_{\mathrm{N}}-\lambda\right)^{-1}
$$

satisfy $s_{k}=\mathrm{O}\left(k^{-\frac{2}{n-1}}\right), k \rightarrow \infty$, and the expression in $(4.15)$ is in $\mathfrak{S}_{p}\left(L^{2}(\Omega)\right)$ for all $p>\frac{n-1}{2}$.

Proof. Lemma 4.7 with $\mathcal{K}=H^{1 / 2}(\partial \Omega), r_{1}=0$ and $r_{2}=\frac{1}{2}$ implies that the embedding operator from $H^{1 / 2}(\partial \Omega)$ into $L^{2}(\partial \Omega)$ belongs to the ideal $\mathfrak{S}_{2(n-1), \infty}\left(H^{1 / 2}(\partial \Omega), L^{2}(\partial \Omega)\right)$. Now Theorem 3.19 with $\mathcal{G}_{0}=\widetilde{\mathcal{G}}_{0}=H^{1 / 2}(\partial \Omega)$ yields that $(4.15)$ is in $\mathfrak{S}_{\frac{2(n-1)}{3}, \infty} \cdot \mathfrak{S}_{2(n-1), \infty}=\mathfrak{S}_{\frac{n-1}{2}, \infty}$, that is, the singular values of (4.15) satisfy $s_{k}=\mathrm{O}\left(k^{-\frac{2}{n-1}}\right)$. Lemma 2.3 (ii) immediately gives the second statement.

By taking differences of resolvent differences, the statements in the next corollary follow directly from Theorems 4.10 and 4.13.

Corollary 4.14. Let $\Theta_{1}$ and $\Theta_{2}$ be self-adjoint relations in $L^{2}(\partial \Omega)$ such that $0 \notin \sigma_{\mathrm{ess}}\left(\Theta_{i}\right)$ and the realizations $A_{\Theta_{i}}, i=1,2$, of $\mathcal{L}$ are self-adjoint operators. Then

$$
\left(A_{\Theta_{1}}-\lambda\right)^{-1}-\left(A_{\Theta_{2}}-\lambda\right)^{-1} \in \mathfrak{S}_{\frac{n-1}{3}, \infty}\left(L^{2}(\Omega)\right)
$$

for all $\lambda \in \rho\left(A_{\Theta_{1}}\right) \cap \rho\left(A_{\Theta_{2}}\right)$ and

$$
\left(A_{\Theta_{1}}-\lambda\right)^{-1}-\left(A_{\mathrm{D}}-\lambda\right)^{-1} \in \mathfrak{S}_{\frac{n-1}{2}, \infty}\left(L^{2}(\Omega)\right)
$$

for all $\lambda \in \rho\left(A_{\Theta_{1}}\right) \cap \rho\left(A_{\mathrm{D}}\right)$.

If the difference $\Theta_{1}-\Theta_{2}$ is itself in some ideal $\mathfrak{S}_{q}$ or $\mathfrak{S}_{q, \infty}$, we obtain an improvement of the first assertion in the previous corollary. For a similar result in a slightly different setting; cf. [14, Theorem 3.7]. 
Theorem 4.15. Let $\Theta_{1}$ and $\Theta_{2}$ be bounded self-adjoint operators in $L^{2}(\partial \Omega)$ such that $0 \notin \sigma_{\mathrm{ess}}\left(\Theta_{i}\right)$ and the realizations $A_{\Theta_{i}}, i=1,2$, of $\mathcal{L}$ from (4.1) are self-adjoint operators. Moreover, assume that $s_{k}\left(\Theta_{1}-\Theta_{2}\right)=\mathrm{O}\left(k^{-1 / q}\right)$, $k \rightarrow \infty$, for some $q>0$. Then, for all $\lambda \in \rho\left(A_{\Theta_{1}}\right) \cap \rho\left(A_{\Theta_{2}}\right)$, the singular values $s_{k}$ of the resolvent difference

$$
\left(A_{\Theta_{1}}-\lambda\right)^{-1}-\left(A_{\Theta_{2}}-\lambda\right)^{-1}
$$

satisfy $s_{k}=\mathrm{O}\left(k^{-\frac{3}{n-1}-\frac{1}{q}}\right), k \rightarrow \infty$, and hence the expression in (4.16) is in $\mathfrak{S}_{p}\left(L^{2}(\Omega)\right)$ for all

$$
p>\frac{q(n-1)}{3 q+n-1} .
$$

Proof. For $\Theta_{1}-\Theta_{2} \in \mathfrak{S}_{q, \infty}$ we conclude from Theorem 3.20 and Proposition 4.8 that the difference of the resolvents in (4.16) is in

$$
\mathfrak{S}_{\frac{2(n-1)}{3}, \infty} \cdot \mathfrak{S}_{q, \infty} \cdot \mathfrak{S}_{\frac{2(n-1)}{3}, \infty}=\mathfrak{S}_{\left(\frac{3}{n-1}+\frac{1}{q}\right)^{-1}, \infty}=\mathfrak{S}_{\frac{q(n-1)}{3 q+n-1}, \infty} .
$$

\subsection{Elliptic operators with $\delta$-interactions on smooth hypersurfaces}

In this section we investigate second order elliptic operators with $\delta$-interactions. Spectral problems for Schrödinger operators with point $\delta$-interactions, as well as $\delta$-interactions on curves and surfaces have been studied extensively during the last decades; see, e.g. [5, 18, 29, 30, 31, 32] and [13], where the same approach as below was used. In order to define self-adjoint elliptic operators in $L^{2}\left(\mathbb{R}^{n}\right)$ with $\delta$-interactions on a smooth compact hypersurface $\Sigma$ in $\mathbb{R}^{n}$ we first construct a suitable quasi boundary triple in Proposition 4.16. One of the main results in this section is Theorem 4.18, where we obtain spectral estimates for the resolvent differences of the operator with a $\delta$-interaction on the hypersurface $\Sigma$ and the unperturbed self-adjoint realization in $L^{2}\left(\mathbb{R}^{n}\right)$.

In the following let $\Omega_{\mathrm{i}} \subset \mathbb{R}^{n}$ be a bounded domain with compact $C^{\infty}$-boundary and let $\Omega_{\mathrm{e}}:=\mathbb{R}^{n} \backslash \overline{\Omega_{\mathrm{i}}}$, so that $\partial \Omega_{\mathrm{i}}=\partial \Omega_{\mathrm{e}}=: \Sigma$ and $\mathbb{R}^{n}=$ $\Omega_{\mathrm{i}} \dot{\cup} \Sigma \dot{\cup} \Omega_{\mathrm{e}}$, and assume that both $\Omega_{\mathrm{i}}$ and $\Omega_{\mathrm{e}}$ are connected. The set $\Omega_{\mathrm{i}}$ is called interior domain and $\Omega_{\mathrm{e}}$ exterior domain. A function $f$ defined on $\mathbb{R}^{n}$ will often be decomposed in the form $f_{\mathrm{i}} \oplus f_{\mathrm{e}}$, where $f_{\mathrm{i}}$ and $f_{\mathrm{e}}$ denote the restrictions of $f$ to the interior and exterior domain, respectively. Let $\mathcal{L}$ be a formally symmetric, uniformly elliptic differential expression as in (4.1) on the Euclidean space $\mathbb{R}^{n}$. The (usual) self-adjoint realization of $\mathcal{L}$ in $L^{2}\left(\mathbb{R}^{n}\right)$ is the operator $A_{\text {free }}$ given by

$$
A_{\text {free }} f=\mathcal{L} f, \quad \operatorname{dom} A_{\text {free }}=\left\{f \in H^{1}\left(\mathbb{R}^{n}\right): \mathcal{L} f \in L^{2}\left(\mathbb{R}^{n}\right)\right\} .
$$

Observe that $A_{\text {free }}$ is the unique self-adjoint operator associated with the quadratic form corresponding to $\mathcal{L}$ on $H^{1}\left(\mathbb{R}^{n}\right)$; cf. [45] and (4.4). The restrictions of $\mathcal{L}$ to the interior domain $\Omega_{\mathrm{i}}$ and exterior domain $\Omega_{\mathrm{e}}$ are denoted by $\mathcal{L}_{\mathrm{i}}$ and $\mathcal{L}_{\mathrm{e}}$, respectively. Clearly, $\mathcal{L}_{\mathrm{i}}$ and $\mathcal{L}_{\mathrm{e}}$ are formally symmetric, uniformly elliptic differential expressions as considered in Sections 4.1 and 4.2. Like in 
Definition 4.1 we introduce the operators $T_{\mathrm{i}}$ and $T_{\mathrm{e}}$ by

$$
\begin{aligned}
T_{\mathrm{i}} f_{\mathrm{i}} & =\mathcal{L}_{\mathrm{i}} f_{\mathrm{i}}, \quad \operatorname{dom} T_{\mathrm{i}}=H^{2}\left(\Omega_{\mathrm{i}}\right), \\
T_{\mathrm{e}} f_{\mathrm{e}} & =\mathcal{L}_{\mathrm{e}} f_{\mathrm{e}}, \quad \operatorname{dom} T_{\mathrm{e}}=\left\{f_{\mathrm{e}} \in H^{1}\left(\Omega_{\mathrm{e}}\right): \mathcal{L}_{\mathrm{e}} f_{\mathrm{e}} \in L^{2}\left(\Omega_{\mathrm{e}}\right),\left.f_{\mathrm{e}}\right|_{\Omega^{\prime}} \in H^{2}\left(\Omega^{\prime}\right)\right\},
\end{aligned}
$$

where $\Omega^{\prime} \subset \Omega_{\mathrm{e}}$ is a bounded subdomain of $\Omega_{\mathrm{e}}$ with smooth boundary such that $\Sigma=\partial \Omega_{\mathrm{e}} \subset \partial \Omega^{\prime}$. The Dirichlet operators on the interior and exterior domain are defined as in Section 4.1 by

$$
\begin{array}{ll}
A_{\mathrm{D}, \mathrm{i}} f_{\mathrm{i}}=\mathcal{L}_{\mathrm{i}} f_{\mathrm{i}}, & \operatorname{dom} A_{\mathrm{D}, \mathrm{i}}=\left\{f_{\mathrm{i}} \in \operatorname{dom} T_{\mathrm{i}}:\left.f_{\mathrm{i}}\right|_{\Sigma}=0\right\}, \\
A_{\mathrm{D}, \mathrm{e}} f_{\mathrm{e}}=\mathcal{L}_{\mathrm{e}} f_{\mathrm{e}}, & \operatorname{dom} A_{\mathrm{D}, \mathrm{e}}=\left\{f_{\mathrm{e}} \in \operatorname{dom} T_{\mathrm{e}}:\left.f_{\mathrm{e}}\right|_{\Sigma}=0\right\} .
\end{array}
$$

Since $A_{\mathrm{D}, \mathrm{i}}$ and $A_{\mathrm{D}, \mathrm{e}}$ are self-adjoint operators, also the orthogonal sum $A_{\mathrm{D}, \mathrm{i}} \oplus$ $A_{\mathrm{D}, \mathrm{e}}$ is a self-adjoint operator in $L^{2}\left(\mathbb{R}^{n}\right)=L^{2}\left(\Omega_{\mathrm{i}}\right) \oplus L^{2}\left(\Omega_{\mathrm{e}}\right)$ and a restriction of the operator $T_{\mathrm{i}} \oplus T_{\mathrm{e}}$. Note that the functions in the domain of $A_{\mathrm{D}, \mathrm{i}} \oplus A_{\mathrm{D}, \mathrm{e}}$ do not necessarily belong to $H^{2}$ in a neighbourhood of $\Sigma$; in general, they belong only to $H^{2}$ in one-sided neighbourhoods of $\Sigma$. In order to treat $\delta$ interactions with quasi boundary triple techniques, we introduce the closed densely defined symmetric operator

$$
A:=A_{\text {free }} \cap\left(A_{\mathrm{D}, \mathrm{i}} \oplus A_{\mathrm{D}, \mathrm{e}}\right)
$$

in $L^{2}\left(\mathbb{R}^{n}\right)$ and the restriction $T$ of $T_{\mathrm{i}} \oplus T_{\mathrm{e}}$ :

$$
T f=\mathcal{L} f, \quad \operatorname{dom} T=\left\{f_{\mathrm{i}} \oplus f_{\mathrm{e}} \in \operatorname{dom}\left(T_{\mathrm{i}} \oplus T_{\mathrm{e}}\right):\left.f_{\mathrm{i}}\right|_{\Sigma}=\left.f_{\mathrm{e}}\right|_{\Sigma}\right\} .
$$

In the next proposition a quasi boundary triple is introduced, which is used to construct operators connected with $\delta$-interactions. The proof is not complicated and uses [10, Theorem 2.3] and is hence omitted. For the case of Schrödinger operators in an $H^{3 / 2}$-setting rather than an $H^{2}$-setting, see [13, Proposition $3.2(\mathrm{i})$ ].

Proposition 4.16. Let $A$ be the closed densely defined symmetric operators in (4.18) and let $T$ be as in (4.19). Then the triple $\left\{L^{2}(\Sigma), \Gamma_{0}, \Gamma_{1}\right\}$, where

$$
\Gamma_{0} \hat{f}=\left.\frac{\partial f_{\mathrm{i}}}{\partial \nu_{\mathcal{L}_{\mathrm{i}}}}\right|_{\Sigma}+\left.\frac{\partial f_{\mathrm{e}}}{\partial \nu_{\mathcal{L}_{\mathrm{e}}}}\right|_{\Sigma} \quad \text { and } \quad \Gamma_{1} \hat{f}=\left.f\right|_{\Sigma}, \quad \hat{f}=\left(\begin{array}{c}
f \\
T f
\end{array}\right), f \in \operatorname{dom} T,
$$

is a quasi boundary triple for $A^{*}$ such that

$$
\operatorname{ker} \Gamma_{0}=A_{\text {free }} \quad \text { and } \quad \operatorname{ker} \Gamma_{1}=A_{\mathrm{D}, \mathrm{i}} \oplus A_{\mathrm{D}, \mathrm{e}}
$$

With the help of the quasi boundary triple from the previous proposition and the operators $A$ and $T$ we define a self-adjoint differential operator $A_{\delta, \alpha}$ associated with $\mathcal{L}$ and $\delta$-interaction with strength $\alpha$ on $\Sigma$. We remark that the operator $A_{\delta, \alpha}$ with a $\delta$-interaction could equivalently be defined with the help of the quadratic form (for the latter see, e.g. [18] or [29]; for the equivalence in the case of a Schrödinger operator see [13, Proposition 3.7]). The statement in the next theorem is essentially a consequence of Theorem 3.11. We remark that in the quasi boundary triple framework also functions $\alpha$ with less smoothness could be allowed. 
Theorem 4.17. Let $\alpha \in C^{1}(\Sigma)$ be real-valued. Then

$$
A_{\delta, \alpha}:=\mathcal{L} \uparrow\left\{\hat{f} \in T: \alpha \Gamma_{1} \hat{f}=\Gamma_{0} \hat{f}\right\}
$$

is a self-adjoint operator in $L^{2}\left(\mathbb{R}^{n}\right)$.

Before proving the theorem we note that the condition $\alpha \Gamma_{1} \hat{f}=\Gamma_{0} \hat{f}$, $\hat{f}=\left(\begin{array}{c}f \\ T f\end{array}\right)$, has the explicit form

$$
\left.\alpha f\right|_{\Sigma}=\left.\frac{\partial f_{\mathrm{i}}}{\partial \nu_{\mathcal{L}_{\mathrm{i}}}}\right|_{\Sigma}+\left.\frac{\partial f_{\mathrm{e}}}{\partial \nu_{\mathcal{L}_{\mathrm{e}}}}\right|_{\Sigma},\left.\quad f_{\mathrm{i}}\right|_{\Sigma}=\left.f_{\mathrm{e}}\right|_{\Sigma}, \quad f=f_{\mathrm{i}} \oplus f_{\mathrm{e}} \in \operatorname{dom} T_{\mathrm{i}} \oplus T_{\mathrm{e}},
$$

and hence one can interpret the operator $A_{\delta, \alpha}$ as an elliptic operator with $\delta$-interaction of strength $\alpha$.

Proof of Theorem 4.17. The quasi boundary triple $\left\{L^{2}(\Sigma), \Gamma_{0}, \Gamma_{1}\right\}$ in Proposition 4.16 satisfies

$$
\operatorname{ran} \Gamma_{0}=H^{1 / 2}(\Sigma) \text { and } \operatorname{ran} \Gamma_{1}=H^{3 / 2}(\Sigma),
$$

so that, for $\lambda \in \rho\left(A_{\mathrm{D}, \mathrm{i}} \oplus A_{\mathrm{D}, \mathrm{e}}\right) \cap \rho\left(A_{\text {free }}\right)$, the corresponding Weyl function $M(\lambda)$ maps $H^{1 / 2}(\Sigma)$ onto $H^{3 / 2}(\Sigma)$. By the same argument as in Lemma 4.4 the closure of $M(\lambda)$ maps $L^{2}(\Sigma)$ into $H^{1}(\Sigma)$, and it follows that this is a compact operator in $L^{2}(\Sigma)$. Since $\alpha h \in H^{1 / 2}(\Sigma)$ for all $h \in H^{1}(\Sigma)$, it follows from Theorem 3.11 with $\Theta=1 / \alpha$ that $A_{\delta, \alpha}$ is self-adjoint.

Now let $\gamma$ be the $\gamma$-field associated with the quasi boundary triple $\left\{L^{2}(\Sigma), \Gamma_{0}, \Gamma_{1}\right\}$ from Proposition 4.16. The same reasoning as in the proof of Proposition 4.8 (i) yields the relation

$$
\gamma(\lambda)^{*} \in \mathfrak{S}_{\frac{2(n-1)}{3}, \infty}\left(L^{2}\left(\mathbb{R}^{n}\right), L^{2}(\Sigma)\right), \quad \lambda \in \rho\left(A_{\text {free }}\right) .
$$

The next theorem is the main result in this subsection. We compare the resolvents of the self-adjoint operator $A_{\delta, \alpha}$ and the unperturbed operator $A_{\text {free. }}$ For Schrödinger operators in an $H^{3 / 2}$-setting, cf. [13, Theorem 4.3].

Theorem 4.18. Let $\alpha \in C^{1}(\Sigma)$ be real-valued. Further, let $A_{\text {free }}$ be the selfadjoint elliptic operator associated with $\mathcal{L}$ in $(4.17)$ and let $A_{\delta, \alpha}$ be the selfadjoint operator from Theorem 4.17. For all $\lambda \in \rho\left(A_{\delta, \alpha}\right) \cap \rho\left(A_{\text {free }}\right)$, the singular values $s_{k}$ of the resolvent difference

$$
\left(A_{\delta, \alpha}-\lambda\right)^{-1}-\left(A_{\text {free }}-\lambda\right)^{-1}
$$

satisfy $s_{k}=\mathrm{O}\left(k^{-\frac{3}{n-1}}\right), k \rightarrow \infty$, and the expression in $(4.21)$ is in $\mathfrak{S}_{p}\left(L^{2}\left(\mathbb{R}^{n}\right)\right)$ for all $p>\frac{n-1}{3}$.

Proof. It follows from Theorem 4.17 that the self-adjoint operator $A_{\delta, \alpha}$ corresponds to the self-adjoint linear relation

$$
\Theta=\left\{\left(\begin{array}{c}
\alpha h \\
h
\end{array}\right): h \in L^{2}(\Sigma)\right\}
$$

via the quasi boundary triple $\left\{L^{2}(\Sigma), \Gamma_{0}, \Gamma_{1}\right\}$, i.e.

$$
A_{\delta, \alpha}=\left\{\hat{f} \in T:\left(\begin{array}{l}
\Gamma_{0} \hat{f} \\
\Gamma_{1} \hat{f}
\end{array}\right) \in \Theta\right\} .
$$


In order to apply Theorem 3.13, we note that the closures of the values of the Weyl function $M(\lambda), \lambda \in \rho\left(A_{\text {free }}\right)$, associated with $\left\{L^{2}(\Sigma), \Gamma_{0}, \Gamma_{1}\right\}$ are compact operators in $L^{2}(\Sigma)$, cf. Lemma 4.4. Since $\alpha$ is assumed to be in $C^{1}(\Sigma)$, it follows that $\Theta^{-1}$ is an everywhere defined bounded operator in $L^{2}(\Sigma)$; in particular, $0 \notin \sigma_{\text {ess }}(\Theta)$. Therefore we can apply Theorem 3.13. Together with (4.20) we conclude that the resolvent difference in (4.21) belongs to

$$
\mathfrak{S}_{\frac{2(n-1)}{3}, \infty} \cdot \mathfrak{S}_{\frac{2(n-1)}{3}, \infty}=\mathfrak{S}_{\frac{n-1}{3}, \infty} .
$$

This shows the statement on the singular values. By Lemma 2.3 (ii) the resolvent difference $(4.21)$ belongs to the classes $\mathfrak{S}_{p}\left(L^{2}\left(\mathbb{R}^{n}\right)\right), p>\frac{n-1}{3}$.

\section{Acknowledgements}

M. Langer was supported by the Engineering and Physical Sciences Research Council (EPSRC) of the UK, grant EP/E037844/1. V. Lotoreichik was supported by the personal grant 2.1/30-04/035 of the government of St. Petersburg and the Leonard Euler programme of DAAD, grant 50077360.

\section{References}

[1] H. Abels, G. Grubb and I. Wood, Extension theory and Krein-type resolvent formulas for nonsmooth boundary value problems, Preprint: arXiv:1008.3281

[2] R. A. Adams and J. J. F. Fournier, Sobolev Spaces, 2nd edition. Pure and Applied Mathematics, vol. 140, Elsevier/Academic Press, Amsterdam, 2003.

[3] M. S. Agranovich, Elliptic operators on closed manifolds, in: Partial differential equations, VI, Encyclopaedia Math. Sci., vol. 63, Springer, Berlin, 1990, pp. 1130.

[4] W. O. Amrein and D. B. Pearson, $M$ operators: a generalisation of WeylTitchmarsh theory, J. Comput. Appl. Math. 171 (2004), 1-26.

[5] J.-P. Antoine, F. Gesztesy and J. Shabani, Exactly solvable models of sphere interactions in quantum mechanics, J. Phys. A 20 (1987), 3687-3712.

[6] W. Arendt and A. F. M. ter Elst, The Dirichlet-to-Neumann operator on rough domains, J. Differential Equations 251 (2011), 2100-2124.

[7] M. S. Ashbaugh, F. Gesztesy, M. Mitrea and G. Teschl, Spectral theory for perturbed Krein Laplacians in nonsmooth domains, Adv. Math. 223 (2010), $1372-1467$.

[8] R. Beals, Non-local boundary value problems for elliptic operators, Amer. J. Math. 87 (1965), 315-362.

[9] J. Behrndt, Elliptic boundary value problems with $\lambda$-dependent boundary conditions, J. Differential Equations 249 (2010), 2663-2687.

[10] J. Behrndt and M. Langer, Boundary value problems for elliptic partial differential operators on bounded domains, J. Funct. Anal. 243 (2007), 536-565.

[11] J. Behrndt and M. Langer, Elliptic operators, Dirichlet-to-Neumann maps and quasi boundary triples, in: Operator Methods for Boundary Value Problems, London Math. Soc. Lecture Note Series, vol. 404, 2012, pp. 121-160. 
[12] J. Behrndt, M. Langer, I. Lobanov, V. Lotoreichik and I. Yu. Popov, A remark on Schatten-von Neumann properties of resolvent differences of generalized Robin Laplacians on bounded domains, J. Math. Anal. Appl. 371 (2010), 750758.

[13] J. Behrndt, M. Langer and V. Lotoreichik, Schrödinger operators with $\delta$ and $\delta^{\prime}$-potentials supported on hypersurfaces, Ann. Henri Poincaré 14 (2013), 385423.

[14] J. Behrndt, M. Langer and V. Lotoreichik, Trace formulae and singular values of resolvent power differences of self-adjoint elliptic operators, J. London Math. Soc. (2), to appear, doi: 10.1112/jlms/jdt012

[15] J. Behrndt, M. M. Malamud and H. Neidhardt, Scattering matrices and Weyl functions, Proc. London Math. Soc. 97 (2008), 568-598.

[16] M. Sh. Birman, Perturbations of the continuous spectrum of a singular elliptic operator by varying the boundary and the boundary conditions, Vestnik Leningrad. Univ. 17 (1962), 22-55 (in Russian); translated in: Amer. Math. Soc. Transl. 225 (2008), 19-53.

[17] M.Sh. Birman and M.Z. Solomjak, Asymptotic behavior of the spectrum of variational problems on solutions of elliptic equations in unbounded domains, Funktsional. Anal. i Prilozhen. 14 (1980), 27-35 (in Russian); translated in: Funct. Anal. Appl. 14 (1981), 267-274.

[18] J. F. Brasche, P. Exner, Yu. A. Kuperin and P. Šeba, Schrödinger operators with singular interactions, J. Math. Anal. Appl. 184 (1994), 112-139.

[19] J. Brasche, M. M. Malamud and H. Neidhardt, Weyl function and spectral properties of selfadjoint extensions, Integral Equations Operator Theory $\mathbf{4 3}$ (2002), 264-289.

[20] B. M. Brown, G. Grubb and I. G. Wood, $M$-functions for closed extensions of adjoint pairs of operators with applications to elliptic boundary problems, Math. Nachr. 282 (2009), 314-347.

[21] B. M. Brown, M. Marletta, S. Naboko and I. Wood, Boundary triplets and $M$-functions for non-selfadjoint operators, with applications to elliptic PDEs and block operator matrices, J. Lond. Math. Soc. (2) 77 (2008), 700-718.

[22] V. M. Bruk, A certain class of boundary value problems with a spectral parameter in the boundary condition, Mat. Sb. (N.S.) 100 (142) (1976), 210-216 (in Russian); translated in: Math. USSR-Sb. 29 (1976), 186-192.

[23] J. Brüning, V. Geyler and K. Pankrashkin, Spectra of self-adjoint extensions and applications to solvable Schrödinger operators, Rev. Math. Phys. 20 (2008), $1-70$.

[24] V. A. Derkach, S. Hassi, M. M. Malamud and H. S. V. de Snoo, Boundary relations and their Weyl families, Trans. Amer. Math. Soc. 358 (2006), 5351-5400.

[25] V.A. Derkach, S. Hassi and H. S. V. de Snoo, Singular perturbations of selfadjoint operators, Math. Phys. Anal. Geom. 6 (2003), 349-384.

[26] V.A. Derkach and M. M. Malamud, Generalized resolvents and the boundary value problems for Hermitian operators with gaps, J. Funct. Anal. 95 (1991), $1-95$.

[27] V.A. Derkach and M. M. Malamud, The extension theory of Hermitian operators and the moment problem, J. Math. Sci. (New York) 73 (1995), 141-242. 
[28] D. E. Edmunds and W. D. Evans, Spectral Theory and Differential Operators. Oxford Mathematical Monographs, The Clarendon Press, Oxford University Press, New York, 1987.

[29] P. Exner, Leaky quantum graphs: a review, in: Analysis on Graphs and its Applications. Selected papers based on the Isaac Newton Institute for Mathematical Sciences programme, Cambridge, UK, 2007. Proc. Symp. Pure Math. 77 (2008), 523-564.

[30] P. Exner and I. Ichinose, Geometrically induced spectrum in curved leaky wires, J. Phys. A 34 (2001), 1439-1450.

[31] P. Exner and S. Kondej, Bound states due to a strong $\delta$ interaction supported by a curved surface, J. Phys. A 36 (2003), 443-457.

[32] P. Exner and K. Yoshitomi, Asymptotics of eigenvalues of the Schrödinger operator with a strong $\delta$-interaction on a loop, J. Geom. Phys. 41 (2002), 344358.

[33] F. Gesztesy and M. Mitrea, Generalized Robin boundary conditions, Robin-toDirichlet maps, and Krein-type resolvent formulas for Schrödinger operators on bounded Lipschitz domains, Proc. Sympos. Pure Math. 79, 105-173, Amer. Math. Soc., 2008.

[34] F. Gesztesy and M. Mitrea, Robin-to-Robin maps and Krein-type resolvent formulas for Schrödinger operators on bounded Lipschitz domains, Oper. Theory Adv. Appl. 191 (2009), 81-113.

[35] F. Gesztesy and M. Mitrea, A description of all self-adjoint extensions of the Laplacian and Krein type resolvent formulas on non-smooth domains, J. Math. Anal. 113 (2011), 53-172.

[36] I. C. Gohberg and M. G. Kreĭn, Introduction to the Theory of Linear Nonselfadjoint Operators. Transl. Math. Monogr., vol. 18., Amer. Math. Soc., Providence, RI, 1969.

[37] V. I. Gorbachuk and M. L. Gorbachuk, Boundary Value Problems for Operator Differential Equations. Kluwer Academic Publishers, Dordrecht, 1991.

[38] P. Grisvard, Elliptic Problems in Nonsmooth Domains. Monographs and Studies in Mathematics, vol. 24, Pitman, Boston, MA, 1985.

[39] G. Grubb, A characterization of the non-local boundary value problems associated with an elliptic operator, Ann. Scuola Norm. Sup. Pisa (3) 22 (1968), $425-513$.

[40] G. Grubb, Singular Green operators and their spectral asymptotics, Duke Math. J. 51 (1984), 477-528.

[41] G. Grubb, Remarks on trace estimates for exterior boundary problems, Comm. Partial Differential Equations 9 (1984), 231-270.

[42] G. Grubb, Distributions and Operators. Graduate Texts in Mathematics, vol. 252, Springer, 2008.

[43] G. Grubb, Spectral asymptotics for Robin problems with a discontinuous coefficient, J. Spectral Theory 1 (2011), 155-177.

[44] T. Kato, Variation of discrete spectra, Comm. Math. Phys. 111 (1987), 501504.

[45] T. Kato, Perturbation Theory for Linear Operators. Springer-Verlag, Berlin, 1995. 
[46] A. N. Kochubei, Extensions of symmetric operators and symmetric binary relations, Math. Zametki 17 (1975), 41-48 (in Russian); translated in: Math. Notes 17 (1975), 25-28.

[47] N. D. Kopachevskiǔ and S. G. Krĕ̌n, Abstract Green formula for a triple of Hilbert spaces, abstract boundary-value and spectral problems, Ukr. Mat. Visn. 1 (2004), 69-97 (in Russian); translated in: Ukr. Math. Bull. 1 (2004), $77-105$.

[48] A. S. Kostenko and M. M. Malamud, 1-D Schrödinger operators with local point interactions on a discrete set, J. Differential Equations 249 (2010), 253-304.

[49] H. Langer and B. Textorius, On generalized resolvents and $Q$-functions of symmetric linear relations (subspaces) in Hilbert space, Pacific J. Math. 72 (1977), $135-165$.

[50] J. Lions and E. Magenes, Non-Homogeneous Boundary Value Problems and Applications I. Springer-Verlag, Berlin-Heidelberg-New York, 1972.

[51] M. M. Malamud, On the formula for generalized resolvents of a non-densely defined Hermitian operator, Ukr. Math. J. 44 (1992), 1522-1547.

[52] M. M. Malamud, Spectral theory of elliptic operators in exterior domains, Russ. J. Math. Phys. 17 (2010), 96-125.

[53] J. Malinen and O. J. Staffans, Impedance passive and conservative boundary control systems, Complex Anal. Oper. Theory 1 (2007), 279-300.

[54] V. Mogilevskiǔ, Boundary triplets and Titchmarsh-Weyl functions of differential operators with arbitrary deficiency indices, Methods Funct. Anal. Topology 15 (2009), 280-300.

[55] V. Mazya, Sobolev Spaces. Springer-Verlag, Berlin, 1985.

[56] A. Pietsch, Operator Ideals. North-Holland Mathematical Library, vol. 20, North-Holland Publishing Co., Amsterdam-New York, 1980.

[57] A. Pietsch, Eigenvalues and s-Numbers. Cambridge Studies in Advanced Mathematics, vol. 13, Cambridge University Press, Cambridge, 1987.

[58] A. Posilicano, Boundary triples and Weyl functions for singular perturbations of self-adjoint operators, Methods Funct. Anal. Topology 10 (2004), 57-63.

[59] A. Posilicano and L. Raimondi, Krein's resolvent formula for self-adjoint extensions of symmetric second-order elliptic differential operators, J. Phys. A 42 (2009), 015204, 11 pp.

[60] O. Post, Spectral Analysis on Graph-Like Spaces. Lecture Notes in Mathematics, vol. 2039, Springer, Heidelberg, 2012.

[61] V. Ryzhov, A general boundary value problem and its Weyl function, Opuscula Math. 27 (2007), 305-331.

[62] V. Ryzhov, Weyl-Titchmarsh function of an abstract boundary value problem, operator colligations, and linear systems with boundary control, Complex Anal. Oper. Theory 3 (2009), 289-322.

[63] R. Seeley, The resolvent of an elliptic boundary problem, Amer. J. Math. 91 (1969), 889-920.

[64] M.I. Vishik, On general boundary problems for elliptic differential equations, Trudy Moskov. Mat. Obšč. 1 (1952), 187-246 (in Russian).

[65] J. Wloka, Partial Differential Equations. Cambridge University Press, Cambridge, 1987. 


\author{
Jussi Behrndt \\ Technische Universität Graz, Institut für Numerische Mathematik, \\ Steyrergasse 30, 8010 Graz, Austria \\ e-mail: behrndt@tugraz.at \\ Matthias Langer \\ Department of Mathematics and Statistics, University of Strathclyde, \\ 26 Richmond Street, Glasgow G1 1XH, United Kingdom \\ e-mail: m.langer@strath.ac.uk \\ Vladimir Lotoreichik \\ Technische Universität Graz, Institut für Numerische Mathematik, \\ Steyrergasse 30, 8010 Graz, Austria \\ e-mail: lotoreichik@math.tugraz.at
}

